\title{
The one-loop effective potential of the Wess-Zumino model revisited
}

\section{Sergei M. Kuzenko and Simon J. Tyler}

School of Physics M013, The University of Western Australia, 35 Stirling Highway, Crawley W.A. 6009, Australia

E-mail: Sergei.Kuzenko@uwa.edu.au, simon.tyler@graduate.uwa.edu.au

ABSTRACT: The full one-loop supersymmetric effective potential for the Wess-Zumino model is calculated using superfield techniques. This includes the Kähler potential and the auxiliary field potential, of which the former was originally computed in 1993 while the latter is derived for the first time. In the purely bosonic sector our results match those of older component field calculations.

In light of prior contradictory results found in the literature, the calculation of the leading term in the auxiliary field potential is approached in a variety of ways. Issues related to conditional convergence that occur during these calculations and their possible consequences are discussed.

KEYwords: Supersymmetric Effective Theories, Renormalization Regularization and Renormalons, Superspaces

ArXiv EPRINT: 1407.5270

In memory of Professor Bruno Zumino 


\section{Contents}

1 Introduction 1

2 Quantization 5

2.1 Propagators of the WZ model 6

$\begin{array}{lll}2.2 & \text { One-loop effective action } & 7\end{array}$

3 Direct expansion of the one-loop effective action $\quad 8$

3.1 Kähler potential $\quad 9$

$\begin{array}{lll}3.2 & \text { Four-derivative term } & 9\end{array}$

4 Auxiliary field potential via the heat kernel 12

$\begin{array}{lll}4.1 \text { Kähler potential } & 12\end{array}$

$\begin{array}{lll}4.2 & \text { Four-derivative term } & 13\end{array}$

$\begin{array}{lll}4.3 & \text { Full auxiliary field potential } & 15\end{array}$

$\begin{array}{lll}5 & \text { Comparisons to the component results } & 17\end{array}$

$\begin{array}{llr}6 & \text { Conclusion and outlook } & 18\end{array}$

A Calculation of the heat kernel $\quad \mathbf{2 0}$

A.1 The differential equations for the heat kernel 20

A.2 Results for the heat kernel 23

$\begin{array}{lll}\text { A.3 Kähler approximation } & 25\end{array}$

\section{Introduction}

The Wess-Zumino (WZ) model was proposed forty years ago $[1,2]$. It is the third oldest supersymmetric field theory in four dimensions. ${ }^{1}$ It is the first off-shell and renormalised supersymmetric field theory ever constructed. As such, it has long acted as both a testbed and a teaching tool for supersymmetry.

We recall that the classical action for the WZ model is ${ }^{2}$

$$
S[\Phi, \bar{\Phi}]=\int \mathrm{d}^{8} z \bar{\Phi} \Phi+\int \mathrm{d}^{6} z \mathcal{P}(\Phi)+\int \mathrm{d}^{6} \bar{z} \overline{\mathcal{P}}(\bar{\Phi}),
$$

where $\mathcal{P}(\Phi)$ denotes the superpotential

$$
\mathcal{P}(\Phi)=\frac{m}{2} \Phi^{2}+\frac{\lambda}{6} \Phi^{3},
$$

\footnotetext{
${ }^{1}$ Only the supersymmetric massive QED of Golfand and Likhtman [3] and the Goldstino model of Volkov and Akulov [4, 5] appeared before the WZ model.

${ }^{2}$ We follow the conventions and notation of $[6]$.
} 
with $m$ and $\lambda$ constant parameters. The dynamical variables are a chiral scalar superfield $\Phi, \bar{D}_{\dot{\alpha}} \Phi=0$, and its complex conjugate $\bar{\Phi}$. The superpotential, $\mathcal{P}(\Phi)$, must be at most cubic for the model to be renormalisable. The mass parameter $m$ can always be chosen to be real and non-negative. The coupling constant $\lambda$ is complex in general. In the massless case, $m=0$, the WZ action is superconformal [1,2].

This paper primarily focuses on the one-loop quantum corrections to the effective potential of the WZ model, in particular, on the auxiliary field potential defined below. In superspace, the full effective action of the WZ model has the generic form [6-8]

$$
\Gamma[\Phi, \bar{\Phi}]=\int \mathrm{d}^{8} z \mathbb{L}\left(\Phi, D_{A} \Phi, \ldots, \bar{\Phi}, D_{A} \bar{\Phi}, \ldots\right)+\left(\int \mathrm{d}^{6} z \mathbb{L}_{c}(\Phi)+\text { c.c. }\right),
$$

where $\mathbb{L}=\bar{\Phi} \Phi+\mathrm{O}(\hbar)$ is the effective superspace Lagrangian, $\mathbb{L}_{c}=\mathcal{P}+\mathrm{O}(\hbar)$ is the effective superpotential, ${ }^{3}$ with $\mathcal{P}(\Phi)$ the classical superpotential. In the first term in the righthand side of (1.3), $D_{A}$ denotes the superspace covariant derivatives, $D_{A}=\left(\partial_{a}, D_{\alpha}, \bar{D}^{\dot{\alpha}}\right)$. For fields constant in spacetime, $\partial_{a} \Phi=\partial_{a} \bar{\Phi}=0$, the effective superspace Lagrangian decomposes into

$$
\left.\mathbb{L}\right|_{\partial_{a} \Phi=\partial_{a} \bar{\Phi}=0}=K(\Phi, \bar{\Phi})+\mathbb{F}\left(\Phi, D_{\alpha} \Phi, D^{2} \Phi, \bar{\Phi}, \bar{D}_{\dot{\alpha}} \bar{\Phi}, \bar{D}^{2} \bar{\Phi}\right),
$$

where

$$
K=\bar{\Phi} \Phi+\sum_{n=1}^{\infty} \hbar^{n} K^{(n)}
$$

is the effective Kähler potential, and

$$
\mathbb{F}=\sum_{n=1}^{\infty} \hbar^{n} \mathbb{F}^{(n)},\left.\quad \mathbb{F}\right|_{D_{\alpha} \Phi=\bar{D}_{\dot{\alpha}} \bar{\Phi}=0}=0,
$$

is called the effective auxiliary field potential (EAFP). The name for $\mathbb{F}$ is appropriate since, when reduced to components in a constant background, the EAFP is of at least third order in the auxiliary fields $[6,8]$. Modulo total derivatives and terms proportional to $\partial_{a} \Phi$ and $\partial_{a} \bar{\Phi}$, the EAFP can always be reduced to the form

$$
\mathbb{F}=\left(D^{\alpha} \Phi\right)\left(D_{\alpha} \Phi\right)\left(\bar{D}_{\dot{\alpha}} \bar{\Phi}\right)\left(\bar{D}^{\dot{\alpha}} \bar{\Phi}\right) \mathbb{G}\left(\Phi, D^{2} \Phi, \bar{\Phi}, \bar{D}^{2} \bar{\Phi}\right) .
$$

This shows that its leading term must have at least four spinor derivatives. The supersymmetric effective potential is determined by $K, \mathbb{F}$ and $\mathbb{L}_{c}$.

\footnotetext{
${ }^{3}$ When all fields are massive, the (chiral) superpotential does not receive any quantum corrections, this was one of the earliest supersymmetric nonrenormalization theorems [2, 9-14]. When there are massless fields present, finite corrections to the superpotential can exist $[15,16]$. In the massless WZ model, the first quantum correction to the superpotential occurs at two loops. It was originally calculated in components by Jack et al. in [17] and then using superfield methods by Buchbinder et al. in [18]. No chiral superpotential is generated at the quantum level if one starts from the effective action for the massive WZ model and then considers a massless limit.
} 
Using the component formulation of the model, the one-loop correction to the effective potential of the WZ model was calculated in the year following the model's proposal [19]. Subsequently this was extended by many authors to include more general models, higher loops and superspace based calculations [20-26]. However, all superspace calculations used a background chiral superfield that did not include a spinor component,

$$
\Phi(\theta)=\phi+\theta^{2} F, \quad \partial_{a} \phi=\partial_{a} F=0,
$$

thus breaking explicit supersymmetry. In all of these papers, the effective potential was always computed as a function of the scalars $\phi, F$ and their conjugates, and never as a superspace Lagrangian of the form (1.4). The point is that, within the standard supergraph technique [14], the problem of computing the quantum corrections to $\mathbb{F}$ is analogous to that of computing quantum corrections with derivatives of fields in ordinary scalar field theories. Actually, in order to determine $\mathbb{F}$, one has to evaluate quantum corrections with an arbitrarily large number of spinor covariant derivatives, which appears to be a daunting task.

The first manifestly supersymmetric calculation of the effective potential of the WZ model was not until $1993[7,8]$. These papers developed a superfield heat kernel technique to compute quantum corrections to the effective potential of the WZ model. The one-loop effective action was expressed in terms of the Green's function for a real scalar superfield in the presence of a background chiral scalar $\Phi$. The heat kernel corresponding to this superpropagator was computed exactly in the case when $\Phi$ satisfies the supersymmetric constraint $\partial_{a} \Phi=0$ and has the explicit form

$$
\Phi(\theta)=\phi+\theta^{\beta} \psi_{\beta}+\theta^{2} F, \quad \partial_{a} \phi=\partial_{a} F=0, \quad \partial_{a} \psi_{\beta}=0 .
$$

Due to the presence of the spinor $\psi_{\beta}$ in the background superfield, the heat kernel derived in $[7,8]$ is significantly more complicated than that which occurs when using the nonsupersymmetric background (1.8).

The heat kernel derived in $[7,8]$ suffices to compute the one-loop supersymmetric effective potential (1.4) exactly, which will be done in this paper. However, explicit calculations were given in $[7,8]$ only for two special structures: the Kähler potential and the leading four-derivative contribution to the EAFP. The one-loop Kähler potential was found to be

$$
K^{(1)}=-\frac{1}{2(4 \pi)^{2}}\left|\mathcal{P}^{\prime \prime}(\Phi)\right|^{2}\left(\ln \frac{\left|\mathcal{P}^{\prime \prime}(\Phi)\right|^{2}}{\mu^{2}}-2\right), \quad \mathcal{P}^{\prime \prime}(\Phi)=m+\lambda \Phi
$$

with $\mu$ the renormalisation scale. The four-derivative correction to $\mathbb{F}$ was found to be

$$
\mathbb{F}_{4 \text {-deriv }}^{(1)}=\zeta \frac{|\lambda|^{4}}{(4 \pi)^{2}} \frac{\left(D^{\alpha} \Phi\right)\left(D_{\alpha} \Phi\right)\left(\bar{D}_{\dot{\alpha}} \bar{\Phi}\right)\left(\bar{D}^{\dot{\alpha}} \bar{\Phi}\right)}{\left|\mathcal{P}^{\prime \prime}(\Phi)\right|^{4}}
$$

for some numerical coefficient $\zeta$. This coefficient was not evaluated explicitly in $[7,8]$, but an integral representation for $\zeta$ was given. In what follows, it will be referred to as $\zeta_{\mathrm{BKY}}$. Since the four-derivative correction (1.11) is UV finite, no regularisation was used in $[7,8]$ for its evaluation. 
The one-loop Kähler potential, $K^{(1)}$, and the leading contribution to the EAFP, $\mathbb{F}_{4 \text {-deriv }}^{(1)}$, were subsequently recalculated using supergraphs [27] and a covariant superfield derivative expansion [28]. Both of these methods are equivalent in spirit to the expansion described in section 3 and would be cumbersome to take to higher orders.

As pointed out in $[7,8]$, the Kähler potential is much easier to calculate than the full supersymmetric effective potential. This is because during the calculation it suffices to use the condition $D_{\alpha} \Phi=0$ for the background field, leading to much simpler propagators. This allows for calculations in more general models and at higher loops [29-31].

Despite the long history described above, many interesting aspects remain to be explored in the calculation of the one-loop supersymmetric effective potential of the WZ model. In this paper we further examine the issues arising in the superfield calculation of the one-loop EAFP. This was motivated by the observation that the direct evaluation of the integral $\zeta_{\mathrm{BKY}}$ given in $[7,8]$ did not match the value of $\zeta$ found in the later papers $[27,28]$, which used dimensional regularisation. We resolve this issue by repeating the earlier calculations and demonstrating that the result is ambiguous due to conditionally convergent integrals. However, using dimensional regularisation fixes the result and yields a coefficient that matches the corresponding term in the earlier component results. We then proceed to use our techniques to present the first superfield calculation of the full one-loop EAFP and compare it to the component results.

Before turning to the computational aspects of this paper, we would like to discuss the functional form of the four-derivative quantum correction (1.11). In the case of the massless WZ model, the expression on the right of (1.11) becomes $\lambda$-independent and proportional to

$$
\frac{\left(D^{\alpha} \Phi\right)\left(D_{\alpha} \Phi\right)\left(\bar{D}_{\dot{\alpha}} \bar{\Phi}\right)\left(\bar{D}^{\dot{\alpha}} \bar{\Phi}\right)}{(\Phi \bar{\Phi})^{2}}=\left(D^{\alpha} \ln \Phi\right)\left(D_{\alpha} \ln \Phi\right)\left(\bar{D}_{\dot{\alpha}} \ln \bar{\Phi}\right)\left(\bar{D}^{\dot{\alpha}} \ln \bar{\Phi}\right) .
$$

For the massless WZ model, it is more advantageous to define the four-derivative quantum correction in a somewhat different form as follows

$$
\widetilde{\mathbb{F}}_{4 \text {-deriv }}^{(1)}=\frac{\zeta}{(4 \pi)^{2}} \Xi,
$$

where we have introduced

$$
\begin{aligned}
\Xi & :=\left[\left(D^{\alpha} \ln \Phi\right)\left(D_{\alpha} \ln \Phi\right)+\left(D^{2} \ln \Phi\right)\right]\left[\left(\bar{D}_{\dot{\alpha}} \ln \bar{\Phi}\right)\left(\bar{D}^{\dot{\alpha}} \ln \bar{\Phi}\right)+\left(\bar{D}^{2} \ln \bar{\Phi}\right)\right] \\
& =\frac{\left(D^{2} \Phi\right)\left(\bar{D}^{2} \bar{\Phi}\right)}{\Phi \bar{\Phi}} .
\end{aligned}
$$

It holds that $\int \mathrm{d}^{8} z \widetilde{\mathbb{F}}_{4 \text {-deriv }}^{(1)} \approx \int \mathrm{d}^{8} z \mathbb{F}_{4 \text {-deriv }}^{(1)}$ modulo the terms proportional to vector derivatives of $\Phi$ and $\bar{\Phi}$. The main advantage of the new definition (1.13a) is that

$$
\left(D^{\alpha} \ln \Phi\right)\left(D_{\alpha} \ln \Phi\right)+\left(D^{2} \ln \Phi\right)=\frac{D^{2} \Phi}{\Phi}
$$

is a (conformal) primary superfield such that the functional $\int \mathrm{d}^{8} z \Xi$ is invariant under the $\mathcal{N}=1$ superconformal transformations (see [6] for a review on $\mathcal{N}=1$ superconformal 
field theories). We recall that the massless WZ model is superconformal at the classical level $[1,2]$. Of course, the superconformal symmetry is anomalous in the quantum theory. However, it is the effective Kähler potential which encodes the information about the superconformal anomaly. It is quite natural to define the EAFP to be superconformal.

In the massless case, the entire one-loop EAFP may be chosen to be a primary superfield of the form

$$
\widetilde{\mathbb{F}}_{\text {massless }}^{(1)}=\Xi \mathbb{H}\left(\frac{\Xi}{\Phi \bar{\Phi}}\right)
$$

for some real function $\mathbb{H}(x)$ such that $\mathbb{H}(0)=\zeta /(4 \pi)^{2}$. It may be seen that $\int \mathrm{d}^{8} z \widetilde{\mathbb{F}}^{(1)}$ is invariant under the superconformal transformations. In accordance with [8], the oneloop effective action of the massless WZ model is invariant under phase transformations $\Phi \rightarrow \mathrm{e}^{\mathrm{i} \tau} \Phi$, with $\tau$ a constant parameter.

The structure of this paper is as follows. In section 2 we examine the quantisation of the WZ model and the structure of its one-loop effective action. In section 3 we use a brute force approach that emulates the diagrammatics of [27] to calculate the one-loop Kähler potential and leading term to the EAFP (1.11). In section 4 we use the heat kernel of appendix A to calculate the one-loop Kähler potential as well as the leading correction and the full expression for the EAFP. The component results for the effective potential and their comparisons to the superfield results are given in section 5. In the last section of this paper summarises the results and looks at the further work that could be done. The paper contains one appendix that repeats the calculation of $[7,8]$ to find the heat kernel for the WZ model. The result is put into the simplest form possible and the Kähler limit is investigated.

Most of the original results given in this work first appeared in the $\mathrm{PhD}$ thesis [32] and many more details can be found in that text and the accompanying auxiliary Mathematica files. Section 2 and appendix A are comprised of review material from [6-8].

\section{Quantization}

The functional integral representation for the effective action $(1.3)$ is $[6,8]$

$$
\mathrm{e}^{\frac{\mathrm{i}}{\hbar} \tilde{\Gamma}[\Phi, \bar{\Phi}]}=\mathcal{N} \int \mathcal{D} \varphi \mathcal{D} \bar{\varphi} \mathrm{e}^{\frac{\mathrm{i}}{\hbar} S^{(\Psi)}[\varphi, \bar{\varphi}]+\mathrm{i} \hbar^{1 / 2} S_{\mathrm{int}}[\varphi, \bar{\varphi}]-\mathrm{i} \hbar^{-1 / 2}\left(\varphi \cdot \frac{\delta \tilde{\Gamma}}{\delta \Phi}+\bar{\varphi} \cdot \frac{\delta \tilde{\Gamma}}{\delta \Phi}\right)},
$$

where $\tilde{\Gamma}[\Phi, \bar{\Phi}]=\Gamma[\Phi, \bar{\Phi}]-S[\Phi, \bar{\Phi}]$ and we have introduced the background chiral scalar

$$
\Psi:=\mathcal{P}^{\prime \prime}(\Phi)=m+\lambda \Phi
$$

and the action

$$
\begin{aligned}
S^{(\Psi)}[\varphi, \bar{\varphi}] & =\int \mathrm{d}^{8} z \bar{\varphi} \varphi+\frac{1}{2}\left(\int \mathrm{d}^{6} z \Psi \varphi^{2}+\text { c.c. }\right), \\
S_{\text {int }}[\varphi, \bar{\varphi}] & =\frac{\lambda}{6} \int \mathrm{d}^{6} z \varphi^{3}+\text { c.c. }
\end{aligned}
$$

From the above, it is clear that the effective action depends on $\Phi$ only through the combination $\Psi$. The only interaction terms in the theory are the cubic vertices of $(2.3 \mathrm{~b})$, however, these are not needed in the one-loop calculations of this paper. 


\subsection{Propagators of the WZ model}

To find the propagators for the model, we note that the Hessian for the free action (2.3a) is defined by

$$
S^{(\Psi)}[\varphi, \bar{\varphi}]=\frac{1}{2}(\varphi, \bar{\varphi}) \cdot H^{(\Psi)} \cdot\left(\begin{array}{c}
\varphi \\
\bar{\varphi}
\end{array}\right), \quad H^{(\Psi)}=\left(\begin{array}{cc}
\Psi & -\frac{1}{4} \bar{D}^{2} \\
-\frac{1}{4} D^{2} & \bar{\Psi}
\end{array}\right)\left(\begin{array}{cc}
\delta_{+} & 0 \\
0 & \delta_{-}
\end{array}\right) .
$$

Where the functional inner product "." is a matrix product as well as the integration over the appropriate superspaces, we suppress the superspace coordinates and use the chiral/antichiral delta function matrix

$$
\left(\begin{array}{cc}
\delta_{+} & 0 \\
0 & \delta_{-}
\end{array}\right)=-\frac{1}{4}\left(\begin{array}{cc}
\bar{D}^{2} & 0 \\
0 & D^{2}
\end{array}\right) \delta^{8}
$$

We can invert the Hessian by writing $G^{(\Psi)}=-H^{(0)} \cdot\left(H^{(\Psi)} \cdot H^{(0)}\right)^{-1}$ and using the block matrix inverse formula to get

$$
G^{(\Psi)}=\left(\begin{array}{cc}
\frac{1}{16} \bar{D}^{2} \frac{1}{\square-\frac{1}{16} \bar{\Psi} D^{2} \frac{1}{\square} \Psi \bar{D}^{2}} \bar{\Psi} \frac{1}{\square} D^{2} & \frac{1}{4} \bar{D}^{2} \frac{1}{\square-\frac{1}{16} \bar{\Psi}^{2} \frac{1}{\square} \Psi \bar{D}^{2}} \\
\frac{1}{4} D^{2} \frac{1}{\square-\frac{1}{16} \Psi \bar{D}^{2} \frac{1}{\square} \bar{\Psi} D^{2}} & \frac{1}{16} D^{2} \frac{1}{\square-\frac{1}{16} \Psi \bar{D}^{2} \frac{1}{\square} \bar{\Psi} D^{2}} \Psi \frac{1}{\square} \bar{D}^{2}
\end{array}\right)\left(\begin{array}{cc}
\delta_{+} & 0 \\
0 & \delta_{-}
\end{array}\right),
$$

where, for the rest of this paper we use the convention that all derivatives act on all terms to the right unless bracketed or otherwise indicated. After using equation (2.5), expanding the inverses as a geometric series and performing some $D$-algebra, the Green's function becomes

$$
G^{(\Psi)}=\frac{1}{16} \sum_{n=0}^{\infty}\left(\begin{array}{ll}
\bar{D}^{2}\left(-\frac{1}{\square} \frac{\bar{\Psi} D^{2}}{-4}-\frac{1}{\square} \frac{\Psi \bar{D}^{2}}{-4}\right)^{n} \bar{D}^{2} & \bar{D}^{2}\left(-\frac{1}{\square} \frac{\bar{\Psi} D^{2}}{-4}-\frac{1}{\square} \frac{\Psi \bar{D}^{2}}{-4}\right)^{n} D^{2} \\
D^{2}\left(-\frac{1}{\square} \frac{\bar{\Psi} D^{2}}{-4}-\frac{1}{\square} \frac{\Psi \bar{D}^{2}}{-4}\right)^{n} \bar{D}^{2} & D^{2}\left(-\frac{1}{\square} \frac{\bar{\Psi} D^{2}}{-4}-\frac{1}{\square} \frac{\Psi \bar{D}^{2}}{-4}\right)^{n} D^{2}
\end{array}\right) \frac{-1}{\square} \delta^{8} .
$$

Resumming the above series recovers the result of [8] and we see that the Green's function can be written in the form

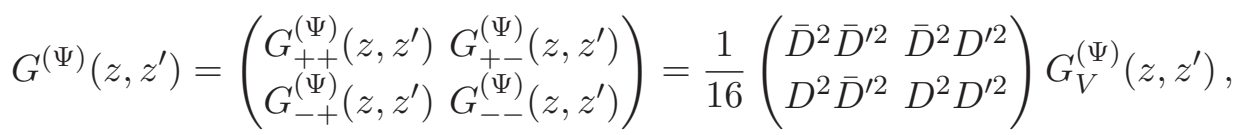

where the auxiliary Green's function $G_{V}^{(\Psi)}$, introduced in [7, 8], satisfies the equation

$$
\Delta G_{V}^{(\Psi)}\left(z, z^{\prime}\right)=-\delta^{8}\left(z, z^{\prime}\right), \quad \text { with } \quad \Delta=\square-\frac{1}{4} \Psi \bar{D}^{2}-\frac{1}{4} \bar{\Psi} D^{2} .
$$

This auxiliary propagator can be understood in terms of its heat kernel representation

$$
\begin{gathered}
G_{V}^{(\Psi)}\left(z, z^{\prime}\right)=\mathrm{i} \int_{0}^{\infty} U_{V}^{(\Psi)}\left(z, z^{\prime} \mid s\right) \mathrm{d} s \\
\left(\partial_{s}-\mathrm{i} \Delta\right) U_{V}^{(\Psi)}\left(z, z^{\prime} \mid s\right)=0, \quad U_{V}^{(\Psi)}\left(z, z^{\prime} \mid 0\right)=\delta^{8}\left(z, z^{\prime}\right) .
\end{gathered}
$$

In the constant background $\partial_{a} \Psi=\partial_{a} \bar{\Psi}=0$ that is the main focus of this paper, the heat kernel factorises to

$$
U_{V}^{(\Psi)}\left(z, z^{\prime} \mid s\right)=\Omega(s) U_{V}^{(0)}\left(z, z^{\prime} \mid s\right)
$$


where $U_{V}^{(0)}\left(z, z^{\prime} \mid s\right)=\delta^{4}\left(\theta-\theta^{\prime}\right) U\left(x, x^{\prime} \mid s\right)$ is the bosonic heat kernel

$$
U\left(x, x^{\prime} \mid s\right)=\frac{-\mathrm{i}}{(4 \pi s)^{2}} \mathrm{e}^{\frac{\mathrm{i}}{4 s}\left(x-x^{\prime}\right)^{2}} .
$$

The operator $\Omega(s)=\mathrm{e}^{-\frac{\mathrm{i} s}{4}\left(\Psi \bar{D}^{2}+\bar{\Psi} D^{2}\right)}$ can be expanded in powers of spinor derivatives

$$
\Omega(s)=\frac{1}{16} A D^{2} \bar{D}^{2}+\frac{1}{16} \tilde{A} \bar{D}^{2} D^{2}+\frac{1}{8} B^{\alpha} D_{\alpha} \bar{D}^{2}+\frac{1}{8} \tilde{B}_{\dot{\alpha}} \bar{D}^{\dot{\alpha}} D^{2}+\frac{1}{4} C D^{2}+\frac{1}{4} \tilde{C} \bar{D}^{2}+1,
$$

where $A, \ldots, \tilde{C}$ are functions of $\partial_{a}, \Psi, D_{\alpha} \Psi,\left(D^{2} \Psi\right)$ and their complex conjugates. The expressions for these functions are derived in detail in appendix A.

\subsection{One-loop effective action}

The one-loop effective action can be written as the functional determinant obtained by turning off the interactions (2.3b) and performing the remaining Gaussian functional integral (2.1) to get

$$
\Gamma^{(1)}=\frac{\mathrm{i}}{2} \log \operatorname{Det}\left(H^{(\Psi)} / H^{(0)}\right)=\frac{\mathrm{i}}{2} \operatorname{Tr} \log \left(H^{(\Psi)} / H^{(0)}\right),
$$

where the functional determinant and trace follow from the inner product defined in (2.4). In particular, the full functional trace decomposes into a trace over the chiral and antichiral subspaces.

$$
\operatorname{Tr}\left(\begin{array}{l}
A_{++} A_{+-} \\
A_{-+}
\end{array} A_{--}\right)=\operatorname{Tr}_{+} A_{++}+\operatorname{Tr}_{-} A_{--}
$$

The argument of the $\log$ in $(2.12)$ is equivalent to

$$
\left(H^{(0)}\right)^{-1} \cdot H^{(\Psi)}=\left(1+\frac{1}{\square}\left(\begin{array}{cc}
0 & -\frac{1}{4} \bar{D}^{2} \bar{\Psi} \\
-\frac{1}{4} D^{2} \Psi & 0
\end{array}\right)\right)\left(\begin{array}{cc}
\delta_{+} & 0 \\
0 & \delta_{-}
\end{array}\right),
$$

and since only the diagonal terms survive the trace (2.13), we obtain

$$
\Gamma^{(1)}=\frac{\mathrm{i}}{4} \operatorname{Tr}_{+} \log \left(1-\frac{\bar{D}^{2}}{4 \square} \bar{\Psi} \frac{D^{2}}{4 \square} \Psi\right)+\text { c.c. }
$$

By using both the the cyclicity of the functional trace and the fact that the trace over chiral superspace is equivalent to the chiral projection ${ }^{4}$ of the trace over full superspace

\footnotetext{
${ }^{4}$ The $\mathcal{N}=1$ superspace projection operators $[33,34]$ are

$$
\begin{aligned}
& P_{+}=\frac{\bar{D}^{2} D^{2}}{16 \square}, \\
& P_{-}=\frac{D^{2} \bar{D}^{2}}{16 \square}, \\
& P_{0}=\frac{D^{\alpha} \bar{D}^{2} D_{\alpha}}{-8 \square}=\frac{\bar{D}_{\dot{\alpha}} D^{2} \bar{D}^{\dot{\alpha}}}{-8 \square}, \\
& P_{0}+P_{+}+P_{-}=1 .
\end{aligned}
$$$$
P_{i} P_{j}=\delta_{i j}
$$ 
$\operatorname{Tr}_{+} F_{++}=\operatorname{Tr}\left(F_{++} P_{+}\right)$, we obtain two useful forms for the one-loop effective action

$$
\begin{aligned}
\Gamma^{(1)} & =\frac{\mathrm{i}}{4} \operatorname{Tr} \sum_{n=1}^{\infty} \frac{-1}{n}\left(\left(P_{+} \bar{\Psi} \frac{1}{\square} \Psi\right)^{n} P_{+}+\text {c.c. }\right) \\
& =\frac{\mathrm{i}}{2} \operatorname{Tr} \sum_{n=1}^{\infty} \frac{-1}{n}\left(\frac{1}{\square} \bar{\Psi} \frac{D^{2}}{4}+\frac{1}{\square} \Psi \frac{\bar{D}^{2}}{4}\right)^{n}=\frac{\mathrm{i}}{2} \operatorname{Tr} \log \left(\frac{\Delta}{\square}\right) .
\end{aligned}
$$

The first form lends itself to a direct expansion of one-loop effective potential performed in section 3, which is similar to the graphical expansion undertaken in [27]. The second form, which can also be derived starting from (2.6), is used for the heat kernel based calculations of $[7,8]$ and section 4 .

All of the above expressions hold in an arbitrary background; however, for the rest of this paper we will primarily focus on the effective potential calculations in a constant background field $\partial_{a} \Psi=\partial_{a} \bar{\Psi}=0$. We find it convenient to use the following notation for the various combinations of derivatives of the background fields

$$
\begin{aligned}
& a=\left(D^{\alpha} \Psi\right)\left(D_{\alpha} \Psi\right), \\
& \bar{a}=\left(\bar{D}_{\dot{\alpha}} \bar{\Psi}\right)\left(\bar{D}^{\dot{\alpha}} \bar{\Psi}\right), \\
& b=\left(D^{2} \Psi\right), \quad \bar{b}=\left(\bar{D}^{2} \bar{\Psi}\right), \\
& u^{2}=\bar{\Psi} \Psi \square, \\
& \mathcal{F}^{2}=\bar{b} b / 64, \\
& \mathcal{G}^{2}=u^{2}+\mathcal{F}^{2} \text {. }
\end{aligned}
$$

For more details, see appendix A.

\section{Direct expansion of the one-loop effective action}

In this section we expand the expression for the one-loop effective action (2.17a) and only keep up to the 4-derivative terms. From (2.17a), we see that we need to examine the term

$$
T_{n}:=\left(P_{+} \bar{\Psi} \frac{1}{\square} \Psi\right)^{n} P_{+},
$$

and its complex conjugate, remembering that all derivatives, unless otherwise indicated, act on all terms to the right. Since we're in the effective potential approximation we can commute all of the $\square^{-1}$ terms to the left.

We're interested in only the Kähler potential and the leading term in the auxiliary potential. To calculate the Kähler potential, we can commute all of the derivatives and therefore all of the projection operators through the background fields to get

$$
T_{n} \approx \square^{-n}(\bar{\Psi} \Psi)^{n} P_{+}
$$

To find the first term in the auxiliary potential, we want to let a total of four Grassmann derivatives hit the backgrounds fields. So, most of the chiral projectors will go straight through to the right; however, there must be a first (from the right) chiral projector that hits a field, so we will need to sum over all possibilities:

$$
T_{n}=\square^{-n} \sum_{j=0}^{n-1}\left(P_{+} \bar{\Psi} \Psi\right)^{n-j-1} \frac{\bar{D}^{2} D^{2}}{16 \square}(\bar{\Psi} \Psi)^{j+1} P_{+} .
$$




\subsection{Kähler potential}

From (2.17a) and (3.2) we see that we can resum the one-loop effective action in the Kähler approximation to get

$$
\Gamma^{(1)}=\int \mathrm{d}^{8} z K^{(1)}=\frac{\mathrm{i}}{4} \operatorname{Tr}\left(\log \left(1-\frac{\bar{\Psi} \Psi}{\square}\right)\left(P_{+}+P_{-}\right)\right) .
$$

Evaluating the trace by moving to momentum space with dimensional regularisation gives the Kähler potential as

$$
K^{(1)}=-\frac{\mathrm{i}}{2} \mu^{2 \varepsilon} \int \frac{\mathrm{d}^{d} k}{(2 \pi)^{d}} \frac{1}{k^{2}} \log \left(1+\frac{\bar{\Psi} \Psi}{k^{2}}\right),
$$

where $d=4-2 \varepsilon$ parametrises the dimensional regularisation scheme and $\mu$ is the minimal subtraction renormalisation mass scale. The integral can be performed to obtain

$$
\begin{aligned}
K^{(1)} & =\frac{1}{2} \mathcal{J}(\bar{\Psi} \Psi) \\
& =\frac{\mu^{2 \varepsilon}}{2(4 \pi)^{2-\varepsilon}} \frac{\Gamma(\varepsilon)}{(1-\varepsilon)^{2}}(\bar{\Psi} \Psi)^{1-\varepsilon}=\frac{\bar{\Psi} \Psi}{2(4 \pi)^{2}}\left(\frac{1}{\varepsilon}+2-\log \frac{\bar{\Psi} \Psi}{\bar{\mu}^{2}}+\mathrm{O}(\varepsilon)\right),
\end{aligned}
$$

where $\bar{\mu}^{2}=4 \pi \mathrm{e}^{-\gamma} \mu^{2}$ is the modified minimal subtraction mass scale. This result agrees with (1.10).

\subsection{Four-derivative term}

The four derivatives in the first projection operator to hit a field in (3.3) can hit the fields in many different ways. Summing over the possibilities we get the terms

$$
\begin{aligned}
T_{n}=\square^{-n} \sum_{j=0}^{n-1}\left(P_{+}\right. & \bar{\Psi} \Psi)^{n-j-1}\left(\left[\bar{D}^{2} D^{2},(\bar{\Psi} \Psi)^{j+1}\right] \frac{1}{16 \square}\right. \\
+ & {\left[\bar{D}^{2} D^{\alpha},(\bar{\Psi} \Psi)^{j+1}\right] \frac{D_{\alpha}}{8 \square}+\left[\bar{D}^{2},(\bar{\Psi} \Psi)^{j+1}\right] \frac{D^{2}}{16 \square} } \\
+ & {\left.\left[\bar{D}^{\dot{\alpha}} D^{\alpha},(\bar{\Psi} \Psi)^{j+1}\right] \frac{\bar{D}_{\dot{\alpha}} D_{\alpha}}{4 \square}+\left[\bar{D}_{\dot{\alpha}},(\bar{\Psi} \Psi)^{j+1}\right] \frac{\bar{D}^{\dot{\alpha}} D^{2}}{8 \square}\right) P_{+} } \\
:=T_{n}^{(1)}+T_{n}^{(2)} & +T_{n}^{(3)}+T_{n}^{(4)}+T_{n}^{(5)} .
\end{aligned}
$$

We will evaluate each term, $T_{n}^{(1, \ldots, 5)}$, in sequence. Note that for $n=1$, only the first term exists, but it is a total derivative and can thus be ignored.

Evaluation of $T_{n}^{(1)}$. Since all four derivatives come from a single $P_{+}$, the rest of the projection operators commute through to the right,

$$
\begin{aligned}
T_{n}^{(1)} & =\frac{1}{\square^{n}} \sum_{j=0}^{n-1} \frac{(\Psi \bar{\Psi})^{n-j-1}}{16 \square}\left[\bar{D}^{2} D^{2},(\Psi \bar{\Psi})^{j+1}\right] P_{+} \\
& =\frac{(\Psi \bar{\Psi})^{n-2}}{16 \square^{n+1}} \sum_{j=0}^{n-1}(j+1)^{2}(\bar{\Psi} \bar{b}+j \bar{a})(\Psi b+j a) P_{+} .
\end{aligned}
$$


Performing the simple sums of polynomials, we find

$$
\begin{aligned}
T_{n}^{(1)}= & \frac{(\Psi \bar{\Psi})^{n-2}}{16 \square^{n+1}} n(n+1)\left(\bar{a} a \frac{(n-1)\left(3 n^{2}-2\right)}{15}\right. \\
& \left.+(\bar{a} \Psi b+a \bar{\Psi} \bar{b}) \frac{(n-1)(3 n+2)}{12}+\bar{\Psi} \bar{b} \Psi b \frac{2 n+1}{6}\right) P_{+} \\
= & \bar{a} a \frac{(\Psi \bar{\Psi})^{n-2}}{16 \square^{n+1}} \frac{n\left(n^{4}-1\right)}{30} P_{+}+\text {surface terms } .
\end{aligned}
$$

Evaluation of $\boldsymbol{T}_{n}^{(2)}$. The first projection operator provides three derivatives to give

$$
T_{n}^{(2)}=\frac{1}{8 \square^{n+1}} \sum_{j=0}^{n-1}(j+1)^{2}\left(P_{+} \bar{\Psi} \Psi\right)^{n-j-1} \bar{\Psi}^{j-1}(\bar{\Psi} \bar{b}+j \bar{a}) \Psi^{j}\left(D^{\alpha} \Psi\right) D_{\alpha} P_{+} .
$$

Since $P_{+} D_{\alpha} P_{+}=0$, the final $D_{\beta}$ to hit a field must come from the next projector on the right. This yields

$$
\begin{aligned}
T_{n}^{(2)}= & -\frac{(\bar{\Psi} \Psi)^{n-2}}{8 \square^{n+1}} \sum_{j=0}^{n-1}(j+1)^{2}(\bar{\Psi} \bar{b}+j \bar{a})(\Psi b+(j+1) a) P_{+} \\
= & \frac{-(\bar{\Psi} \Psi)^{n-2}}{8 \square^{n+1}} \frac{n(n+1)}{60}\left(\bar{a} a(n-1)\left(12 n^{2}+15 n+2\right)+5 \bar{a} \Psi b(n-1)(3 n+2)\right. \\
& \quad+15 a \bar{\Psi} \bar{b} n(n+1)+10 \bar{\Psi} \bar{b} \Psi b(2 n+1)) P_{+} \\
= & -\bar{a} a \frac{(\bar{\Psi} \Psi)^{n-2}}{8 \square^{n+1}} \frac{(n-2)(n-1) n(n+1)(2 n-1)}{60} P_{+}+\text {surface terms } .
\end{aligned}
$$

Evaluation of $\boldsymbol{T}_{n}^{(3)}$. Although only two derivatives come from the first $P_{+}$, because $P_{+} D^{2} P_{+}=0$ the rest of the derivatives must come from the next projection operator, so the evaluation of $T_{n}^{(3)}$ is very similar to $T_{n}^{(2)}$. The result is

$$
\begin{aligned}
T_{n}^{(3)}= & \frac{(\bar{\Psi} \Psi)^{n-2}}{16 \square \square^{n+1}} \frac{n(n+1)(n+2)}{60}(3 \bar{a} a(n-1)(4 n+2)+15 \bar{a} \Psi b(n-1) \\
& +5 a \bar{\Psi} \bar{b}(3 n+1)+20 \bar{\Psi} \bar{b} \Psi b) P_{+} \\
= & \bar{a} a \frac{(\bar{\Psi} \Psi)^{n-2}}{16 \square^{n+1}} \frac{(n-2)(n-1) n(n+1)(n+2)}{30} P_{+}+\text {surface terms } .
\end{aligned}
$$

Evaluation of $\boldsymbol{T}_{n}^{(4)}$. One $D_{\alpha}$ and one $\bar{D}_{\dot{\alpha}}$ from the first projection operator hit fields leaving

$$
T_{n}^{(4)}=\frac{1}{4 \square^{n+1}} \sum_{j=0}^{n-1}(j+1)^{2}\left(P_{+} \bar{\Psi} \Psi\right)^{n-j-1}(\bar{\Psi} \Psi)^{j}\left(\bar{D}^{\dot{\alpha}} \bar{\Psi}\right)\left(D^{a} \Psi\right) \bar{D}_{\dot{\alpha}} D_{\alpha} P_{+}
$$


Since $\bar{D}_{\dot{\alpha}} D_{\alpha} P_{+}=-2 \mathrm{i} \partial_{\alpha \dot{\alpha}} P_{+}$, the next derivative can come from any of the remaining projection operators. We sum over all possibilities and, after some work get the result

$$
\begin{aligned}
T_{n}^{(4)} & =-a \frac{(\bar{\Psi} \Psi)^{n-2}}{8 \square^{n+1}} \sum_{j=0}^{n-1} \sum_{k=0}^{n-j-2}(j+1)^{2}(\bar{\Psi} \bar{b}+(j+k+1) \bar{a}) P_{+} \\
& =-a \frac{(\bar{\Psi} \Psi)^{n-2}}{8 \square^{n+1}} \frac{(n-1) n(n+1)}{12}\left(n \bar{\Psi} \bar{b}+\frac{\bar{a}}{10}\left(8 n^{2}-5 n-2\right)\right) P_{+} \\
& =\bar{a} a \frac{(\bar{\Psi} \Psi)^{n-2}}{8 \square^{n+1}} \frac{(n-2)(n-1) n(n+1)(2 n-1)}{120} P_{+}+\text {surface terms }
\end{aligned}
$$

Evaluation of $\boldsymbol{T}_{\boldsymbol{n}}^{(\mathbf{5})}$. The evaluation of $T_{n}^{(5)}$ is similar to that of $T_{n}^{(4)}$, the final result is

$$
\begin{aligned}
T_{n}^{(5)}=- & \frac{(\bar{\Psi} \Psi)^{n-2}}{8 \square^{n+1}} \frac{n(n+1)(n+2)}{60}\left(\bar{a} a\left(16 n^{2}-13 n-3\right)\right. \\
& +5 \bar{a} \Psi b(3 n+1)+20 \bar{\Psi} \bar{b} a(n-1)+20 \bar{\Psi} \bar{b} \Psi b) P_{+} \\
=- & \bar{a} a \frac{(\bar{\Psi} \Psi)^{n-2}}{8 \square^{n+1}} \frac{(n-2)(n-1) n(n+1)(n+2)}{60} P_{+}+\text {surface terms . }
\end{aligned}
$$

Total. Combining all of the above, we find that

$$
\begin{aligned}
T_{n}=-\frac{(\bar{\Psi} \Psi)^{n-2}}{16 \square^{n+1}} & \frac{n(n+1)}{12}\left(\bar{a} a\left(8 n^{3}+5 n^{2}-11 n-2\right)\right. \\
& \left.+2 \bar{a} \Psi b\left(3 n^{2}+5 n+4\right)+2 a \bar{\Psi} \bar{b}\left(5 n^{2}+3 n-8\right)+2 \bar{\Psi} \bar{b} \Psi b(4 n+5)\right) P_{+},
\end{aligned}
$$

which becomes remarkably simple after integration by parts

$$
T_{n}=\bar{a} a \frac{(\bar{\Psi} \Psi)^{n-2}}{16 \square^{n+1}} \frac{n^{2}\left(n^{2}-1\right)}{12} P_{+}+\text {surface terms } .
$$

We can now calculate the 4-derivative correction to the EAFP

$$
\Gamma_{4 \text {-deriv }}^{(1)}=\frac{\mathrm{i}}{4} \operatorname{Tr} \sum_{n=1}^{\infty} \frac{-1}{n}\left(T_{n} P_{+}+\text {c.c. }\right) \text {. }
$$

Using the expression of $T_{n}$ after integration by parts (3.9) and moving to momentum space to diagonalise the trace, we have

$$
\Gamma_{4 \text {-deriv }}^{(1)}=\frac{1}{(4 \pi)^{2}} \int \mathrm{d}^{8} z \frac{\bar{a} a}{32} \int_{0}^{\infty} \mathrm{d} k \frac{k^{3}}{\left(k^{2}+\bar{\Psi} \Psi\right)^{4}} .
$$

Performing the final momentum integral yields a result of the form $\Gamma_{4 \text {-deriv }}^{(1)}=\int \mathrm{d}^{8} z \mathbb{F}_{4 \text {-deriv }}$, where $\mathbb{F}_{4 \text {-deriv }}$ is given by (1.11) with

$$
\zeta=\frac{1}{384},
$$

in agreement with the calculations of [27] and [28].

If, instead, we use (3.8), then, provided we integrate by parts before performing the momentum integral, we obtain the same result. However, if we leave the integration by 
parts until last, then each of the four terms in the momentum integral are IR divergent. In which case, the momentum integrals can be performed if, e.g., we regularise with dimensional regularisation. The result is

$$
\begin{aligned}
\Gamma_{4 \text {-deriv }}^{(1)}=\frac{\left(4 \pi \mu^{2}\right)^{\varepsilon}}{\Gamma(2-\varepsilon)(4 \pi)^{2}} \int \mathrm{d}^{8} z \frac{1 / 96}{(\bar{\Psi} \Psi)^{2}}\left(\frac{\bar{a} a}{2}\left(\frac{1}{\varepsilon}-\log (\bar{\Psi} \Psi)-\frac{13}{2}\right)\right. \\
\left.+(\bar{a} \Psi b+\text { c.c. })\left(\frac{1}{\varepsilon}-\log (\bar{\Psi} \Psi)+1\right)-\frac{\bar{\Psi} \bar{b} \Psi b}{2}\left(\frac{5}{\varepsilon}-5 \log (\bar{\Psi} \Psi)-9\right)\right) .
\end{aligned}
$$

Integrating by parts, the $\frac{1}{\varepsilon}$ and log terms cancel and we once again recover the result (1.11) with $\zeta$ given by $(3.11)$.

\section{Auxiliary field potential via the heat kernel}

In this section we examine the one-loop effective action of the WZ model starting with its expression in terms of the heat kernel $U_{V}^{(\Psi)}(s)$ studied in appendix A. As shown in [7, 8], the one-loop effective action may be represented in the form

$$
\Gamma^{(1)}=\frac{\mathrm{i}}{2} \operatorname{Tr} \log \left(\frac{\Delta}{\square}\right)=-\frac{\mathrm{i}}{2} \operatorname{Tr} \log \left(G_{V}^{(\Psi)}\right)=-\frac{\mathrm{i}}{2} \int_{0}^{\infty} \frac{\mathrm{d} s}{s} \operatorname{Tr} U_{V}^{(\Psi)}(s) .
$$

In the effective potential limit, where $\partial_{a} \Phi=\partial_{a} \bar{\Phi}=0$, it reduces to

$$
\Gamma^{(1)}=-\left.\frac{\mathrm{i}}{2} \int \mathrm{d}^{8} z \int_{0}^{\infty} \frac{\mathrm{d} s}{s}(A(s)+\tilde{A}(s)) U\left(x, x^{\prime} \mid s\right)\right|_{x^{\prime} \rightarrow x},
$$

which is the sum of the Kähler and auxiliary potentials

$$
\Gamma^{(1)}=\int \mathrm{d}^{8} z\left(K^{(1)}+\mathbb{F}^{(1)}\right) .
$$

In the following subsections we evaluate the Kähler potential to check the above and to establish some notation. We then evaluate the four-derivative term in the auxiliary potential, first using the integral introduced in $[7,8]$

$$
\mathbb{J}(s):=\frac{2}{s} \int_{0}^{\infty} \sin (p) \mathrm{e}^{-p^{2} / s} \mathrm{~d} p=\sqrt{\frac{\pi}{s}} \mathrm{e}^{-s / 4} \operatorname{erfi}\left(\frac{\sqrt{s}}{2}\right),
$$

and then using other methods to show that the term is actually conditionally convergent. Finally we use the lessons learnt in the previous subsections to evaluate the full auxiliary potential.

\subsection{Kähler potential}

In the Kähler approximation (see section A.3) the diagonal of the heat kernel reduces to

$$
\begin{aligned}
U_{V}^{(\Psi)}(z, z \mid s) & =\left.(\cos s u-1)\left(P_{+}+P_{-}\right) \delta^{4}\left(\theta-\theta^{\prime}\right) U\left(x, x^{\prime} \mid s\right)\right|_{z^{\prime} \rightarrow z} \\
& =\left.2 \bar{\Psi} \Psi \frac{\cos s u-1}{u^{2}} U\left(x, x^{\prime} \mid s\right)\right|_{x^{\prime} \rightarrow x},
\end{aligned}
$$


where $u^{2}=\bar{\Psi} \Psi \square$ and $U\left(x, x^{\prime} \mid s\right)$ is the dimensionally regularised bosonic heat kernel defined in (A.25).

The proper-time integral in (4.1) can then be evaluated by first moving to momentum space. After Wick rotating and integrating out the angular parts this leads to the following expression for the Kähler potential

$$
K^{(1)}=\frac{-\mu^{2 \varepsilon}}{(4 \pi)^{2-\varepsilon}} \frac{2}{\Gamma(2-\varepsilon)} \int_{0}^{\infty} \int_{0}^{\infty} k^{1-2 \varepsilon}(\cos (s k|\Psi|)-1) \mathrm{e}^{-k^{2} s} \mathrm{~d} k \mathrm{~d} s .
$$

The remaining integrals can be performed in either order to get the result

$$
K^{(1)}=\frac{1}{2} \mathcal{J}(\bar{\Psi} \Psi)=\frac{\bar{\Psi} \Psi}{2(4 \pi)^{2}}\left(\frac{1}{\varepsilon}+2-\log \frac{\bar{\Psi} \Psi}{\bar{\mu}^{2}}+\mathrm{O}(\varepsilon)\right),
$$

which matches (3.6).

Alternatively, we can follow $[7,8]$ and swap dimensional regularisation for a propertime cutoff $s_{0} \rightarrow 0$. Performing the momentum integral in (4.6) now gives

$$
K^{(1)}=\frac{\Psi \bar{\Psi}}{2(4 \pi)^{2}} \int_{\mathrm{i} s_{0}}^{\infty} \frac{\mathrm{d} s}{s} \mathbb{J}(s \Psi \bar{\Psi})
$$

where $\mathbb{J}$ is defined in (4.4). This integral can be evaluated in terms of a hypergeometric function and expanded around $s_{0}=0$ to give

$$
K^{(1)}=\frac{\Psi \bar{\Psi}}{2(4 \pi)^{2}}\left(-\log \left(\mathrm{i} s_{0} \mu^{2} \mathrm{e}^{\gamma}\right)+2-\log \left(\frac{\bar{\Psi} \Psi}{\mu^{2}}\right)+\mathrm{O}\left(s_{0}\right)\right)
$$

for some renormalisation scale $\mu^{2}$.

\subsection{Four-derivative term}

To find the coefficient of the leading term in the auxiliary potential, we need to evaluate (4.2) keeping only the four-derivative terms in the expression for $A(s)+\tilde{A}(s)$. Expanding the results of section A.2 gives

$$
\begin{aligned}
A(s)+\left.\tilde{A}(s)\right|_{4 \text {-deriv }}= & \frac{s \bar{a} a}{512 u^{3}}\left(\left(7-\frac{10}{3} s^{2} u^{2}\right) \sin (s u)+s u\left(s^{2} u^{2}-7\right) \cos (s u)\right) \\
& +\frac{s(\bar{\Psi} \bar{b} a+\text { c.c. })}{64 u^{3}}\left(s u \cos (s u)-\left(1-\frac{s^{2} u^{2}}{3}\right) \sin (s u)\right) \\
& +\frac{s \bar{\Psi} \bar{b} \Psi b}{64 u^{3}}(\sin (s u)-s u \cos (s u))
\end{aligned}
$$

A general term in (4.9) is of the form $\mathbb{A}=s u^{-3} \mathbf{A}(s u)$ and its contribution to the effective potential (4.2) is

$$
\left.\Gamma^{(1)}\right|_{\mathbb{A}}=-\left.\frac{\mathrm{i}}{2} \int \mathrm{d}^{8} z \int_{0}^{\infty} \frac{\mathrm{d} s}{s} \frac{s^{4}}{(s u)^{3}} \mathbf{A}(s u) U\left(x, x^{\prime} \mid s\right)\right|_{x^{\prime} \rightarrow x} .
$$


By using the $d$-dimensional momentum space representation for $U\left(x, x^{\prime} \mid s\right)$, eq. (A.25), then integrating out the angular parts of the momentum integral, Wick rotating and rescaling the proper-time integral, we obtain

$$
\left.\Gamma^{(1)}\right|_{\mathbb{A}}=\frac{\mu^{2 \varepsilon}}{\Gamma(2-\varepsilon)(4 \pi)^{d / 2}} \int \frac{\mathrm{d}^{8} z}{(\bar{\Psi} \Psi)^{2+\varepsilon}} \int_{0}^{\infty} \frac{\mathrm{d} s}{s^{1-2 \varepsilon}} \int_{0}^{\infty} \frac{\mathrm{d} q}{q^{2 \varepsilon}} \mathbf{A}(q) \mathrm{e}^{-\frac{q^{2}}{s}},
$$

where we've defined $q=s|k| \sqrt{\bar{\Psi} \Psi}$.

Removing the dimensional regularisation, it is now straightforward to use the definition (4.4) in order to perform the momentum integral in (4.11) to write the four derivative contribution as

$$
\begin{aligned}
\Gamma_{4 \text {-deriv }}^{(1)}=\frac{1}{64(4 \pi)^{2}} \int \frac{\mathrm{d}^{8} z}{(\bar{\Psi} \Psi)^{2}} \int_{0}^{\infty} \frac{\mathrm{d} s}{s}\left(\frac{s \bar{\Psi} \bar{b} \Psi b}{4}((s+2) \mathbb{J}(s)-2)\right. & \\
- & \frac{s(\bar{\Psi} \bar{b} a+\text { c.c. })}{24}\left(\left(s^{2}+4 s+12\right) \mathbb{J}(s)-2(s+6)\right) \\
+ & \left.\frac{s \bar{a} a}{384}\left(\left(3 s^{3}+2 s^{2}+44 s+168\right) \mathbb{J}(s)-2\left(3 s^{2}+8 s+84\right)\right)\right) .
\end{aligned}
$$

Each of the three terms in the above proper-time integral are IR divergent, but the divergences cancel when combined using integration by parts. This gives a result of the form

$$
\mathbb{F}_{4 \text {-deriv }}=\zeta \frac{\left(D^{\alpha} \Phi\right)\left(D_{\alpha} \Phi\right)\left(\bar{D}_{\dot{\alpha}} \bar{\Phi}\right)\left(\bar{D}^{\dot{\alpha}} \bar{\Phi}\right)}{(4 \pi)^{2}|m+\lambda \Phi|^{4}}
$$

where $\zeta=\zeta_{\mathrm{BKY}}$ is defined by the integral

$$
\zeta_{\mathrm{BKY}}=\frac{1}{1024} \int_{0}^{\infty} \mathrm{d} s\left(1-\mathbb{J}(s)+\frac{s}{2}(\mathbb{J}(s)+4)-\frac{s^{2}}{4}(5 \mathbb{J}(s)+1)+\frac{s^{3}}{8} \mathbb{J}(s)\right) .
$$

Up to some typographical errors, this matches equation (5.15) of $[7,8]$. This integral can be evaluated to give the numerical result $\zeta_{\mathrm{BKY}}=-\frac{1}{64}$, which clearly does not match the value of $\zeta=\frac{1}{384}$ found in the previous section.

Alternatively if we first integrate (4.9) by parts to get the expression

$$
A(s)+\left.\tilde{A}(s)\right|_{4 \text {-deriv }} \approx \frac{s \bar{a} a}{1536 u^{3}}\left(3\left(1+2 s^{2} u^{2}\right) \sin (s u)-\left(3-s^{2} u^{2}\right) s u \cos (s u)\right),
$$

which holds up to surface terms, we can then evaluate (4.11) without regularisation, as in the last paragraph, to find the four-derivative correction (1.11) with

$$
\zeta=\frac{1}{1024} \int_{0}^{\infty} \mathrm{d} s\left(\mathbb{J}(s)-1+\frac{s}{2}\left(3 \mathbb{J}(s)+\frac{8}{3}\right)-\frac{s^{2}}{4}\left(3 \mathbb{J}(s)+\frac{1}{3}\right)+\frac{s^{3}}{8} \frac{1}{3} \mathbb{J}(s)\right) .
$$

This result is different from (4.12) and evaluates to the numerical value of $\frac{1}{192}$ which agrees with neither of the previously found values for $\zeta$.

The problem lies in the fact that the unregularised $(\varepsilon \rightarrow 0)$ integrals are only conditionally convergent and not invariant under the rescaling required to obtain (4.11). If 
we don't perform the rescaling then it is convenient to try exchanging the order of the proper-time and momentum integrals, as it leads to simpler intermediate expressions that are free from the error functions $\mathbb{J}(s)$. However, when the order of the unregularised integrals is exchanged the result of the integration changes. This is a clear sign of conditional convergence.

If we keep the dimensional regularisation used in (4.11) then we consistently get the correction (1.11) with $\zeta=\frac{1}{384}$. We demonstrate this with two possible order of operations. First, we start with (4.13) and perform the proper-time integral to get

$$
\Gamma_{4 \text {-deriv }}^{(1)}=\frac{\mu^{2 \varepsilon}}{\Gamma(2-\varepsilon)(4 \pi)^{d / 2}} \int \mathrm{d}^{8} z \frac{\bar{a} a}{32} \int_{0}^{\infty} \mathrm{d} k \frac{k^{3-2 \varepsilon}}{\left(k^{2}+\bar{\Psi} \Psi\right)^{4}} .
$$

This momentum integral is clearly equivalent to (3.10) and converges for $-2<\varepsilon<2$, so it does not need dimensional regularisation. We recover the result (1.11) with $\zeta=\frac{1}{384}$. However, if we start with (4.9) and leave the integration by parts until the very end, then we definitely need the dimensional regularisation. Once again, for simplicity, performing the proper-time integral first, we find

$$
\begin{aligned}
\Gamma_{4 \text {-deriv }}^{(1)}=\frac{\left(4 \pi \mu^{2}\right)^{\varepsilon}}{\Gamma(2-\varepsilon)(4 \pi)^{2}} \int \mathrm{d}^{8} z \frac{\bar{\Psi} \Psi}{8} \int_{0}^{\infty} \frac{\mathrm{d} k}{k^{1+2 \varepsilon}}\left(\frac{\bar{a} a}{12} \frac{5 \bar{\Psi} \Psi-4 k^{2}}{\left(k^{2}+\bar{\Psi} \Psi\right)^{4}}\right. \\
\left.-\frac{a \bar{\Psi} \bar{b}+\text { c.c. }}{3\left(k^{2}+\bar{\Psi} \Psi\right)^{3}}+\frac{b \bar{b}}{4\left(k^{2}+\bar{\Psi} \Psi\right)^{2}}\right) .
\end{aligned}
$$

The momentum integrals are IR divergent (i.e., in dimensional regularisation, they converge for $-2<\varepsilon<0)$ and we get the $\varepsilon$-expansion

$$
\begin{aligned}
\Gamma_{4 \text {-deriv }}^{(1)}=\frac{\left(4 \pi \mu^{2}\right)^{\varepsilon}}{\Gamma(2-\varepsilon)(4 \pi)^{2}} \int \mathrm{d}^{8} z \frac{1 / 96}{(\bar{\Psi} \Psi)^{2}}\left(\frac{\bar{a} a}{2}\left(-\frac{5}{\varepsilon}+5 \log (\bar{\Psi} \Psi)-\frac{21}{2}\right)\right. \\
\left.+(a \bar{\Psi} \bar{b}+\text { c.c. })\left(\frac{2}{\varepsilon}-2 \log (\bar{\Psi} \Psi)+3\right)-\frac{3 \bar{\Psi} \bar{b} \Psi b}{2}\left(\frac{1}{\varepsilon}-\log (\bar{\Psi} \Psi)+1\right)\right) .
\end{aligned}
$$

This looks similar to (3.12), however the coefficients of the terms are different. Nevertheless, integrating by parts yields the same (1.11) with $\zeta=\frac{1}{384}$.

We note that in $[7,8]$, the action of $A(s)+\tilde{A}(s)$ on $U\left(x, x^{\prime} \mid s\right)$ was not evaluated by going to momentum space, but rather by series expansion and using (A.4). This leads to essentially identical results and problems to those discussed above. See the auxiliary Mathematica documents in [32] for more details of this calculation and for other calculations using different regularisation schemes.

\subsection{Full auxiliary field potential}

In the previous subsections, we have seen that the most robust and compact way to calculate the leading correction to the auxiliary potential is to use the dimensionally regularised heat kernel, integrate by parts first, then perform the proper-time integral and finally the momentum space integral. We will now follow this procedure to calculate the full oneloop EAFP. 
The first step is to use integration by parts to get $A(s)+\tilde{A}(s)$ into a usable form. Starting with the results (A.23) we find, after some work,

$$
\begin{aligned}
\Psi C(s)+\bar{\Psi} \tilde{C}(s) \approx-2 \mathrm{i} \bar{\Psi} \Psi & \frac{\sin (s u)}{u}-\mathrm{i} \overline{\bar{b} b}\left(\left(\frac{s^{2} u^{2}-1}{2 u}-\frac{u}{\mathcal{F}^{2}}\right) \sin (s u)\right. \\
& \left.-\frac{3 s}{2} \cos (s u)+\frac{\mathcal{G}}{\mathcal{F}}\left(\frac{\cos (s \mathcal{F}) \sin (s \mathcal{G})}{\mathcal{F}}+\frac{\sin (s \mathcal{F}) \cos (s \mathcal{G})}{\mathcal{G}}\right)\right),
\end{aligned}
$$

which can then be integrated using (A.9a) to get

$$
\begin{aligned}
A(s)+\tilde{A}(s) \approx 2 \bar{\Psi} \Psi & \frac{\cos (s u)-1}{u} \\
& +\frac{\bar{a} a}{\bar{b} b}\left(\frac{s^{2}}{2}\left(\cos (s u)+\frac{\sin (s u)}{s u}\right)+\frac{\cos (s \mathcal{F}) \cos (s \mathcal{G})-\cos (s u)}{\mathcal{F}^{2}}\right) .
\end{aligned}
$$

The first term is derivative free and corresponds to the Kähler approximation discussed above. The second term contains all of the terms that generate the auxiliary potential, starting with four derivative term (4.13).

Equation (4.14) is an amazingly simple expression, considering the complexity of the results found in appendix $\mathrm{A}$, and is quite easily integrated to give the low-energy effective action. The general structure is

$$
\Gamma^{(1)}=\frac{\mu^{2 \varepsilon}(4 \pi)^{-d / 2}}{\Gamma(2-\varepsilon)} \int \mathrm{d}^{8} z \int_{0}^{\infty} \mathrm{d} k k^{3-2 \varepsilon} \int_{0}^{\infty} \frac{\mathrm{d} s}{s}(A(-\mathrm{i} s, u)+\tilde{A}(-\mathrm{i} s, u)) \mathrm{e}^{-k^{2} s} .
$$

Performing the proper-time integral yields

$$
\begin{aligned}
\Gamma^{(1)}=\frac{\mu^{2 \varepsilon}(4 \pi)^{-d / 2}}{\Gamma(2-\varepsilon)} \int \mathrm{d}^{8} z \int_{0}^{\infty} \mathrm{d} k k^{3-2 \varepsilon}\left[2 \bar{\Psi} \Psi \frac{\log \left(1+\bar{\Psi} \Psi / k^{2}\right)}{2 k^{2} \bar{\Psi} \Psi}\right. \\
\left.+\frac{\bar{a} a}{\bar{b} b}\left(\frac{-1}{\left(k^{2}+\bar{\Psi} \Psi\right)^{2}}+\frac{2 \log \left(\frac{\bar{\Psi} \Psi}{k^{2}}+1\right)-\log \left(\left(\frac{\bar{\Psi} \Psi}{k^{2}}+1\right)^{2}-\frac{4 \mathcal{F}^{2}}{k^{2}}\right)}{4 \mathcal{F}^{2}}\right)\right] .
\end{aligned}
$$

Factorising the final logarithm term, the momentum integral can then be evaluated to get

$$
\begin{aligned}
\Gamma^{(1)}=\frac{\mu^{2 \varepsilon} \Gamma(\varepsilon)}{(4 \pi)^{d / 2} \Gamma(2-\varepsilon)} & \int \mathrm{d}^{8} z\left[\frac{\Gamma(1-\varepsilon)}{2(1-\varepsilon)}(\bar{\Psi} \Psi)^{1-\varepsilon}-\frac{\bar{a} a}{2 \bar{b} b}\left(\frac{\Gamma(2-\varepsilon)}{(\bar{\Psi} \Psi)^{\varepsilon}}\right.\right. \\
& \left.\left.+\frac{\Gamma(1-\varepsilon)}{(2-\varepsilon)} \frac{2(\bar{\Psi} \Psi)^{2-\varepsilon}-(\bar{\Psi} \Psi+2 \mathcal{F})^{2-\varepsilon}-(\bar{\Psi} \Psi-2 \mathcal{F})^{2-\varepsilon}}{4 \mathcal{F}^{2}}\right)\right] .
\end{aligned}
$$

Expanding around $d=4$ and simplifying we get our result

$$
\Gamma^{(1)}=\int \mathrm{d}^{8} z\left(K^{(1)}+\mathbb{F}^{(1)}\right),
$$

where the Kähler potential $K^{(1)}$ was given in (3.6) and the EAFP is

$$
\begin{aligned}
& (4 \pi)^{2} \mathbb{F}^{(1)}=\frac{1}{4} \frac{\bar{a} a}{\bar{b} b}\left(3-\left(1+\frac{16 \bar{\Psi}^{2} \Psi^{2}}{\bar{b} b}\right) \log \left(1-\frac{\bar{b} b}{16 \bar{\Psi}^{2} \Psi^{2}}\right)\right. \\
& \left.-\frac{16 \bar{\Psi} \Psi}{\sqrt{\bar{b} b}} \operatorname{coth}^{-1}\left(\frac{4 \bar{\Psi} \Psi}{\sqrt{\bar{b} b}}\right)\right) \text {. }
\end{aligned}
$$


This has the series expansion

$$
\begin{aligned}
(4 \pi)^{2} \mathbb{F}^{(1)} & =\frac{\bar{a} a}{4} \sum_{n=1}^{\infty} \frac{1}{n(n+1)(2 n+1)} \frac{(\bar{b} b)^{n-1}}{(4 \bar{\Psi} \Psi)^{2 n}} \\
& =\frac{\bar{a} a}{\bar{\Psi}^{2} \Psi^{2}}\left(\frac{1}{384}+\frac{1}{30720} \frac{\bar{b} b}{(\bar{\Psi} \Psi)^{2}}+\frac{1}{1376256} \frac{(\bar{b} b)^{2}}{(\bar{\Psi} \Psi)^{4}}+\ldots\right),
\end{aligned}
$$

where the natural expansion parameter is the dimensionless

$$
p^{2}=\frac{\bar{b} b}{(4 \bar{\Psi} \Psi)^{2}}
$$

Using integration by parts to remove $\bar{a} a$ from the EAFP essentially requires that we integrate $F^{(1)}$ with respect to $p$ twice. This yields an expression with dilogarithms

$$
\begin{aligned}
(4 \pi)^{2} \mathbb{F}^{(1)}= & \frac{1}{4} \sum_{n=1}^{\infty} \frac{1}{n(n+1)(2 n+1)(2 n-1)^{2}} \frac{(\bar{b} b)^{n}}{(4 \bar{\Psi} \Psi)^{2 n}} \\
= & \frac{\bar{\Psi} \Psi}{36}\left(8+3 p \operatorname{Li}_{2}(p)-3 p \operatorname{Li}_{2}(-p)\right. \\
& \left.\quad-\frac{1}{2 p^{2}}\left((p+1)\left(11 p^{2}+7 p+2\right) \log (p+1)+(p \rightarrow-p)\right)\right) .
\end{aligned}
$$

This is reminiscent of $[28,30,35]$ where, for a $\mathcal{N}=2$ SYM theory written in terms of $\mathcal{N}=1$ superfields, the one-loop Kähler potential was twice integrated to recover the $\mathcal{N}=2$ nonholomorphic potential. Their results were also expressed using dilogarithms.

\section{Comparisons to the component results}

Note that in the above two sections, the complicated and often poorly behaved expressions simplified enormously after unifying the various terms through integration by parts. In the component form of the effective potential with the background

$$
\Phi=\phi+\theta^{2} f, \quad \partial \phi=\partial f=0,
$$

the functional forms are unique. ${ }^{5}$ This makes the component expressions a lot simpler to work with than their superfield counterparts.

The calculation of the full one-loop effective potential for the Wess-Zumino model has be performed many times before [19-26] and does not need to be repeated here. We will just quote the results, see [32] for more details. The effective potential can be written as the dimensionally regularised momentum space integral

$$
V^{(1)}=\frac{\mathrm{i}}{2} \int \frac{\mathrm{d}^{d} k}{(2 \pi)^{d}} \log \left(1-\frac{|\lambda f|^{2}}{\left(k^{2}+\left|m^{\prime}\right|^{2}\right)^{2}}\right),
$$

\footnotetext{
${ }^{5}$ If instead of working with the effective potential, we are interested in the effective action, then the functional forms are once again not unique due to integration by parts identities in the spacetime integrals.
} 
where $m^{\prime}=\left.\Psi\right|_{\theta=\bar{\theta}=0}=m+\lambda \phi$. The momentum integral can be performed and yields

$$
\begin{aligned}
(4 \pi)^{2} V^{(1)}= & \frac{|\lambda f|^{2}}{2}\left(-\frac{1}{\varepsilon}+\log \frac{\left|m^{\prime}\right|^{2}}{\bar{\mu}^{2}}+\mathrm{O}(\varepsilon)\right) \\
& -|\lambda f|^{2}\left(\frac{3}{4}-\frac{\left|m^{\prime}\right|^{2}}{|\lambda f|} \tan ^{-1} \frac{|\lambda f|}{\left|m^{\prime}\right|^{2}}-\frac{1}{4}\left(1+\frac{\left|m^{\prime}\right|^{4}}{|\lambda f|^{2}}\right) \log \left(1-\frac{|\lambda f|^{2}}{\left|m^{\prime}\right|^{4}}\right)\right) .
\end{aligned}
$$

Remembering that $\Gamma^{(1)}=-\int \mathrm{d}^{4} x V^{(1)}$, we see that the Kähler potential in superspace projects to give the first line in (5.2) through the relation

$$
\int \mathrm{d}^{8} z K(\Phi, \bar{\Phi})=\int \mathrm{d}^{4} x \bar{f} f \partial_{\phi} \partial_{\bar{\phi}} K(\phi, \bar{\phi}) .
$$

Equally as straightforward, the first line in (5.2) can be lifted to superspace to give the Kähler potential by a simple double integral.

The derivative expansion of the EAFP is expressed using the dimensionless quantity $p^{2}$ defined in (4.18). It projects to the same ratio of component fields seen in $(5.2 \mathrm{~b})$

$$
\left.p^{2}\right|_{\Phi=\phi+\theta^{2} f}=p_{\mid}^{2}=\left|\frac{\lambda f}{m^{\prime 2}}\right|^{2} .
$$

Given the EAFP in the form $\bar{\Psi} \Psi f\left(p^{2}\right)$, it can easily be projected to components using

$$
\int \mathrm{d}^{8} z \bar{\Psi} \Psi f\left(p^{2}\right)=\int \mathrm{d}^{4} x|\lambda f|^{2}\left(1-p_{\mid} \partial_{p_{\mid}}\right)^{2} f\left(p_{\mid}^{2}\right) .
$$

Equivalently, the component expression for the EAFP in the form $|\lambda f|^{2} g\left(p_{\mid}^{2}\right)$ can be lifted to the superfield expression

$$
\mathbb{F}^{(1)}(\Phi, \bar{\Phi}, a, \bar{a}, b, \bar{b})=\frac{\bar{a} a}{(4 \bar{\Psi} \Psi)^{2}} \frac{g\left(p^{2}\right)}{p^{2}} .
$$

Either way, we see that the EAFP given in (4.16) and (5.2b) are equivalent.

\section{Conclusion and outlook}

In this paper we have completed the calculation started in $[7,8]$ and used superfield techniques to compute the full one-loop supersymmetric effective potential for the WZ model. This includes both the effective Kähler potential and the previously unpublished result for the EAFP. In the purely bosonic sector our results match the older component results for the effective potential of the WZ model.

The supersymmetric effective potential contains more information than the ordinary effective potential of the WZ model. The point is that the superfield expressions (3.6) and (4.16) also generate two- and four-fermionic contributions (generalised Yukawa couplings). Of course, once the most general functional structure of the supersymmetric effective potential is known, one can read off the expressions for $K$ and $\mathbb{F}$ from the component results. However, this functional structure became available as a result of the superfield heat kernel calculation of $[7,8]$. 
We have also compared different methods for calculating the leading term in the EAFP and accounted for the different results in the literature by noting that the calculation includes conditionally convergent integrals. We observed that dimensional regularisation removes the conditional convergence and gives results that agree with the corresponding term in the component calculations. It is interesting to observe that apparently finite terms in the effective action can result from conditionally convergent integrals in some calculation schemes. This leads to possible ambiguities in calculations that can not be fixed by renormalisation conditions like those in nominally divergent terms. One of the authors (SJT) has observed a similar problem occur in a two-loop effective action calculation in $\mathcal{N}=2$ super-Yang-Mills. How to identify when such cases can occur and how to fix the ambiguity is an open question.

The calculation of the leading term in the EAFP had possible IR divergences before the different structures were unified through integration by parts, while the full EAFP does not appear to have such a problem and seems to not suffer any conditional convergence. However, the expressions in the EAFP calculation are more unwieldy and this hinders their exploration. The momentum integrals in the EAFP are also difficult to perform as a whole and we used dimensional regularisation to handle the UV divergences that appeared when treating them separately. The issue of any possible conditional convergence and ambiguity in the full EAFP is not completely closed. Although, we should note that some of the component results were calculated using other regularisation schemes and they yielded consistent results that match that which we presented in (4.16).

In calculating the EAFP at the one-loop level, we used the highly simplified structure of the heat kernel that occurs after integration by parts. However, in higher loop calculations the full structure of the heat kernel is needed. This would make it an interesting challenge to try to carry out the superfield calculation of the EAFP to two loops. Alternatively, the calculation could be performed with the background (1.8) and that result lifted to superspace using the results in the above section. There are three published component calculations of the two-loop effective potential of the WZ model [26, 36-38]. However the results of [36] were left as unevaluated Feynman integrals and the results in [26, 37, 38] contain terms that are less than quadratic in the auxiliary fields and thus can not come from the projection of a superfield action. As for two-loop superfield calculations, both the effective Kähler potential and the effective chiral potential have been calculated many times, but the EAFP does not appear in the literature. It would be good to have a definitive result for it and the component effective potential at two loops.

In the massless case, as discussed in section 1, the one-loop EAFP of the massless WZ model can be chosen in the form (1.14) such that the functional $\int \mathrm{d}^{8} z \widetilde{\mathbb{F}}^{(1)}$ is superconformal. This naturally leads us to consider a higher-derivative extension of the massless WZ model that is superconformal. It is described by an action of the form

$$
S[\Phi, \bar{\Phi}]=\int \mathrm{d}^{8} z \bar{\Phi} \Phi\{1+\bar{\Sigma} \Sigma \mathfrak{H}(\Sigma, \bar{\Sigma})\}+\lambda \int \mathrm{d}^{6} z \Phi^{3}+\bar{\lambda} \int \mathrm{d}^{6} \bar{z} \bar{\Phi}^{3},
$$

where $\mathfrak{H}(w, \bar{w})$ is a real analytic function and the chiral scalar

$$
\Sigma:=\Phi^{-2} \bar{D}^{2} \bar{\Phi}
$$


is a (conformal) primary superfield of dimension zero. ${ }^{6}$ The massless WZ model possesses a $\mathbb{Z}_{3}$ symmetry generated by $\Phi \rightarrow \mathrm{e}^{\frac{2}{3} \pi \mathrm{i}} \Phi$. This symmetry remains intact for the higherderivative extension of the WZ model given by (6.1), since $\Sigma$ is invariant under the $\mathbb{Z}_{3}$ group. In the massless case, the one-loop effective action is invariant under $\mathrm{U}(1)$ phase transformations $\Phi \rightarrow \mathrm{e}^{\mathrm{i} \tau} \Phi$. Requiring this symmetry gives $\mathfrak{H}(\Sigma, \bar{\Sigma})=\hat{\mathfrak{H}}(\Sigma \bar{\Sigma})$. It would be interesting to see whether the U(1) invariant functional form of the EAFP, eq. (1.14), survives at two loops.

\section{A Calculation of the heat kernel}

The superfield heat kernel (2.8) was computed in [8] in the constant background case, $\partial_{a} \Psi=\partial_{a} \bar{\Psi}=0$. The original derivation presented in [8] contained some typographical errors. In this appendix we provide a corrected and simplified derivation of the heat kernel. This is essential for our new results in section 4 .

\section{A.1 The differential equations for the heat kernel}

As we saw in section 2, all propagators that occur in the Wess-Zumino model with arbitrary background chiral superfields $\Psi$ and $\bar{\Psi}$ can be obtained as different chiral projections of the Green's function of the operator

$$
\Delta=\square-\frac{1}{4}\left(\Psi \bar{D}^{2}+\bar{\Psi} D^{2}\right) .
$$

The heat kernel of this operator obeys the differential equation (DE) and initial condition

$$
\begin{aligned}
\left(\mathrm{i} \frac{\mathrm{d}}{\mathrm{d} s}+\Delta\right) U_{V}^{(\Psi)}\left(z, z^{\prime} \mid s\right) & =0 \\
U_{V}^{(\Psi)}\left(z, z^{\prime} \mid 0\right) & =\delta^{4}\left(\theta-\theta^{\prime}\right) \delta^{4}\left(x-x^{\prime}\right) .
\end{aligned}
$$

If we assume that the background is constant over space-time, $\partial_{a} \Psi=\partial_{a} \bar{\Psi}=0$, then the heat kernel factorises as $[7,8]$

$$
U_{V}^{(\Psi)}\left(z, z^{\prime} \mid s\right)=\Omega(s) U_{V}^{(0)}\left(z, z^{\prime} \mid s\right), \quad \Omega(s):=\mathrm{e}^{-\frac{\mathrm{i} s}{4}\left(\bar{\Psi} D^{2}+\Psi \bar{D}^{2}\right)},
$$

where $U_{V}^{(0)}\left(z, z^{\prime} \mid s\right)=\delta^{4}\left(\theta-\theta^{\prime}\right) U\left(x, x^{\prime} \mid s\right)$,

$$
\square U\left(x, x^{\prime} \mid s\right)=-\mathrm{i} \frac{\partial}{\partial s} U\left(x, x^{\prime} \mid s\right),
$$

and

$$
\begin{aligned}
U\left(x, x^{\prime} \mid s\right) & =\exp (\mathrm{i} s \square) \delta^{4}\left(x-x^{\prime}\right) \\
& =\int \frac{\mathrm{d}^{4} k}{(2 \pi)^{4}} \mathrm{e}^{-\mathrm{i} k^{2} s+\mathrm{i} k\left(x-x^{\prime}\right)}=-\frac{\mathrm{i}}{(4 \pi s)^{2}} \mathrm{e}^{\frac{\mathrm{i}}{4}\left(x-x^{\prime}\right)^{2} / s},
\end{aligned}
$$

is the free bosonic heat kernel. To find the full heat kernel (A.3) we need only obtain an explicit form of the operator $\Omega(s)$.

\footnotetext{
${ }^{6}$ The superfield $\Xi$ defined by (1.13b) can be represented as $\Xi=\bar{\Phi} \Phi \bar{\Sigma} \Sigma$.
} 
The heat equation (A.1) implies that the operator $\Omega(s)$ satisfies

$$
\mathrm{i} \frac{\mathrm{d}}{\mathrm{d} s} \Omega(s)=\frac{1}{4} \Omega(s)\left(\Psi \bar{D}^{2}+\bar{\Psi} D^{2}\right), \quad \Omega(0)=1 .
$$

To solve this, following $[7,8]$, we expand the operator $\Omega(s)$ as

$$
\begin{aligned}
\Omega(s)=\frac{1}{16} A(s) D^{2} \bar{D}^{2}+\frac{1}{16} \tilde{A}(s) \bar{D}^{2} D^{2}+\frac{1}{8} B^{\alpha}(s) D_{\alpha} \bar{D}^{2} & +\frac{1}{8} \tilde{B}_{\dot{\alpha}}(s) \bar{D}^{\dot{\alpha}} D^{2} \\
& +\frac{1}{4} C(s) D^{2}+\frac{1}{4} \tilde{C}(s) \bar{D}^{2}+1 .
\end{aligned}
$$

Note that only $A$ and $\tilde{A}$ can contribute to the 1-loop potential. At this point, it is convenient to introduce some notation

$$
\begin{aligned}
& a=\left(D^{\alpha} \Psi\right)\left(D_{\alpha} \Psi\right), \quad b=\left(D^{2} \Psi\right), \quad \mu=\left(D^{\alpha} \Psi\right)\left(\bar{D}^{\dot{\alpha}} \bar{\Psi}\right) \partial_{\alpha \dot{\alpha}}, \\
& u^{2}=\bar{\Psi} \Psi \square, \quad \mathcal{F}^{2}=\bar{b} b / 64, \quad \mathcal{G}^{2}=u^{2}+\mathcal{F}^{2}, \quad \beta=\frac{1}{8}\left(\begin{array}{ll}
0 & \bar{b} \\
b & 0
\end{array}\right) .
\end{aligned}
$$

We can move between the tilded and non-tilded symbols in (A.7) by making the replacements $D^{\alpha} \leftrightarrow \bar{D}_{\dot{\alpha}}, \Psi \leftrightarrow \bar{\Psi}$ which imply that $a \leftrightarrow \bar{a}, b \leftrightarrow \bar{b}$ and $\mu \leftrightarrow-\mu$. Since we are using the convention that derivatives act on all terms to their right unless bracketed, $\mu$ is actually a differential operator that obeys $\mu^{2}=-\frac{1}{2} \bar{a} a \square$.

With the above expansion, the heat equation (A.6) decomposes as

$$
\begin{aligned}
\frac{\mathrm{d}}{\mathrm{d} s}\left(\begin{array}{c}
A \\
\tilde{A}
\end{array}\right) & =-\mathrm{i}\left(\begin{array}{cc}
\Psi & 0 \\
0 & \bar{\Psi}
\end{array}\right)\left(\begin{array}{c}
C \\
\tilde{C}
\end{array}\right), \\
\left(\frac{\mathrm{d}}{\mathrm{d} s}+\left(\begin{array}{cc}
0 & \Psi \partial^{\dot{\alpha} \alpha} \\
\bar{\Psi} \partial_{\alpha \dot{\alpha}} & 0
\end{array}\right)\right)\left(\begin{array}{c}
B^{\alpha} \\
\tilde{B}_{\dot{\alpha}}
\end{array}\right) & =-\mathrm{i}\left(\begin{array}{c}
\left(D^{\alpha} \Psi\right) C \\
\left(\bar{D}_{\dot{\alpha}} \bar{\Psi}\right) \tilde{C}
\end{array}\right), \\
\left(\frac{\mathrm{d}}{\mathrm{d} s}+2 \mathrm{i} \beta\right)\left(\begin{array}{c}
C \\
\tilde{C}
\end{array}\right)+\mathrm{i}\left(\begin{array}{c}
\bar{\Psi}(\square A+1) \\
\Psi(\square \tilde{A}+1)
\end{array}\right) & =-\frac{1}{2}\left(\begin{array}{c}
B^{\alpha} \partial_{\alpha \dot{\alpha}}\left(\bar{D}^{\dot{\alpha}} \bar{\Psi}\right) \\
\tilde{B}_{\dot{\alpha}} \partial^{\dot{\alpha} \alpha}\left(D_{\alpha} \Psi\right)
\end{array}\right),
\end{aligned}
$$

with $A(0)=\tilde{A}(0)=B^{\alpha}(0)=\tilde{B}_{\dot{\alpha}}(0)=C(0)=\tilde{C}(0)=0$. We can eliminate $A$ and $\tilde{A}$ from the equation for $C$ and $\tilde{C}$ by moving to the second order DE

$$
\left(\frac{\mathrm{d}^{2}}{\mathrm{~d} s^{2}}+2 \mathrm{i} \beta \frac{\mathrm{d}}{\mathrm{d} s}+\bar{\Psi} \Psi \square\right)\left(\begin{array}{c}
C \\
\tilde{C}
\end{array}\right)=-\frac{1}{2} \frac{\mathrm{d}}{\mathrm{d} s}\left(\begin{array}{c}
B^{\alpha} \partial_{\alpha \dot{\alpha}}\left(\bar{D}^{\dot{\alpha}} \bar{\Psi}\right) \\
\tilde{B}_{\dot{\alpha}} \partial^{\alpha \dot{\alpha}}\left(D_{\alpha} \Psi\right)
\end{array}\right),
$$

where we need the initial "velocity" $\left.\partial_{s}(C, \tilde{C})\right|_{s=0}=-\mathrm{i}(\bar{\Psi}, \Psi)$.

We solve the coupled equations (A.9b), (A.9d) for $B$ and $C$ by expanding with respect to the Grassmann parameters $D_{\alpha} \Psi$ and $\bar{D}_{\dot{\alpha}} \bar{\Psi}$,

$$
\begin{aligned}
C & =C_{0}+a C_{20}+\bar{a} C_{02}+\mu C_{11}+\bar{a} a C_{22}, \\
\tilde{C} & =\tilde{C}_{0}+\bar{a} \tilde{C}_{20}+a \tilde{C}_{02}-\mu \tilde{C}_{11}+\bar{a} a \tilde{C}_{22}, \\
B^{\alpha} & =\left(D^{\alpha} \Psi\right)\left(\hat{B}_{0}+\bar{a} \hat{B}_{2}\right)+\left(\bar{D}_{\dot{\alpha}} \bar{\Psi}\right) \partial^{\dot{\alpha} \alpha}\left(\check{B}_{0}+a \check{B}_{2}\right), \\
\tilde{B}_{\dot{\alpha}} & =\left(\bar{D}_{\dot{\alpha}} \bar{\Psi}\right)\left(\hat{\tilde{B}}_{0}+a \hat{\tilde{B}}_{2}\right)+\left(D^{\alpha} \Psi\right) \partial_{\alpha \dot{\alpha}}\left(\check{\tilde{B}}_{0}+\bar{a} \overline{\tilde{B}}_{2}\right) .
\end{aligned}
$$


This expansion is used to find a system of ordinary second order differential equations for $B$ and $C$. For the rest of this subsection, we simply extract and list the DEs order by order in $D_{\alpha} \Psi$ and $\bar{D}_{\dot{\alpha}} \bar{\Psi}$. In the next subsection we note the common structure to the DEs and provide their solutions.

Keeping all terms independent of $D_{\alpha} \Psi$ and $\bar{D}_{\dot{\alpha}} \bar{\Psi}$ in (A.9d) gives the homogeneous second order DE

$$
\left(\frac{\mathrm{d}^{2}}{\mathrm{~d} s^{2}}+2 \mathrm{i} \beta \frac{\mathrm{d}}{\mathrm{d} s}+u^{2}\right)\left(\begin{array}{l}
C_{0} \\
\tilde{C}_{0}
\end{array}\right)=0
$$

with the initial conditions

$$
\left.\left(\begin{array}{c}
C_{0} \\
\tilde{C}_{0}
\end{array}\right)\right|_{s=0}=0,\left.\quad \frac{\mathrm{d}}{\mathrm{d} s}\left(\begin{array}{c}
C_{0} \\
\tilde{C}_{0}
\end{array}\right)\right|_{s=0}=-\mathrm{i}\left(\begin{array}{c}
\bar{\Psi} \\
\Psi
\end{array}\right) .
$$

Keeping only the first order terms in (A.9b) gives

$$
\left(\begin{array}{c}
\mathrm{d} \\
\mathrm{d} s
\end{array}+\left(\begin{array}{cc}
0 & \Psi \partial^{\alpha \dot{\alpha}} \\
\bar{\Psi} \partial_{\alpha \dot{\alpha}} & 0
\end{array}\right)\right)\left(\begin{array}{c}
\left(D^{\alpha} \Psi\right) \hat{B}_{0}+\left(\bar{D}_{\dot{\alpha}} \bar{\Psi}\right) \partial^{\alpha \dot{\alpha}} \check{B}_{0} \\
\left(\bar{D}_{\dot{\alpha}} \bar{\Psi}\right) \hat{\tilde{B}}_{0}+\left(D^{\alpha} \Psi\right) \partial_{\alpha \dot{\alpha}} \tilde{\tilde{B}}_{0}
\end{array}\right)=-\mathrm{i}\left(\begin{array}{c}
\left(D^{\alpha} \Psi\right) C_{0} \\
\left(\bar{D}_{\dot{\alpha}} \bar{\Psi}\right) \tilde{C}_{0}
\end{array}\right) .
$$

Extracting the coefficients of $D_{\alpha} \Psi$ and $\bar{D}_{\dot{\alpha}} \bar{\Psi}$ leads to two equations that can be recombined to give the second order DE for $\check{B}_{0}$

$$
\left(\frac{\mathrm{d}^{2}}{\mathrm{~d} s^{2}}+u^{2}\right)\left(\begin{array}{c}
\check{B}_{0} \\
\tilde{B}_{0}
\end{array}\right)=\mathrm{i}\left(\begin{array}{cc}
0 & \Psi \\
\bar{\Psi} & 0
\end{array}\right)\left(\begin{array}{c}
C_{0} \\
\tilde{C}_{0}
\end{array}\right)
$$

the solution of which immediately gives $\hat{B}_{0}$ through the relation

$$
\left(\begin{array}{c}
\hat{B}_{0} \\
\hat{\tilde{B}}_{0}
\end{array}\right)=\frac{-1}{\bar{\Psi} \Psi}\left(\begin{array}{cc}
0 & \Psi \\
\bar{\Psi} & 0
\end{array}\right) \frac{\mathrm{d}}{\mathrm{d} s}\left(\begin{array}{c}
\check{B}_{0} \\
\tilde{\tilde{B}}_{0}
\end{array}\right) .
$$

Keeping only the second order terms in (A.9d) yields the two second order DEs

$$
\begin{aligned}
\left(\frac{\mathrm{d}^{2}}{\mathrm{~d} s^{2}}+2 \mathrm{i} \beta \frac{\mathrm{d}}{\mathrm{d} s}+u^{2}\right)\left(\begin{array}{c}
a C_{20}+\bar{a} C_{02} \\
\bar{a} \tilde{C}_{20}+a \tilde{C}_{02}
\end{array}\right) & =\frac{\square}{2} \frac{\mathrm{d}}{\mathrm{d} s}\left(\begin{array}{c}
\bar{a} \check{B}_{0} \\
a \tilde{B}_{0}
\end{array}\right), \\
\left(\frac{\mathrm{d}^{2}}{\mathrm{~d} s^{2}}+2 \mathrm{i} \beta \frac{\mathrm{d}}{\mathrm{d} s}+u^{2}\right)\left(\begin{array}{c}
C_{11} \\
-\tilde{C}_{11}
\end{array}\right) & =-\frac{1}{2} \frac{\mathrm{d}}{\mathrm{d} s}\left(\begin{array}{c}
\hat{B}_{0} \\
\hat{\tilde{B}}_{0}
\end{array}\right) .
\end{aligned}
$$

Although we could separate the DEs for $C_{20}$ and $C_{02}$, it is simpler (more symmetric) to solve for them simultaneously.

Keeping only the third order terms in (A.9b) gives

$$
\begin{aligned}
\left(\frac{\mathrm{d}}{\mathrm{d} s}+\left(\begin{array}{cc}
0 & \Psi \partial^{\dot{\alpha} \alpha} \\
\bar{\Psi} \partial_{\alpha \dot{\alpha}} & 0
\end{array}\right)\right) & \left(\begin{array}{l}
\bar{a}\left(D^{\alpha} \Psi\right) \hat{B}_{2}+a\left(\bar{D}_{\dot{\beta}} \bar{\Psi}\right) \partial^{\dot{\beta} \alpha} \check{B}_{2} \\
a\left(\bar{D}_{\dot{\alpha}} \bar{\Psi}\right) \hat{\tilde{B}}_{2}+\bar{a}\left(D^{\beta} \Psi\right) \partial_{\beta \dot{\alpha}} \tilde{\tilde{B}}_{2}
\end{array}\right) \\
& =-\mathrm{i}\left(\begin{array}{c}
\left(D^{\alpha} \Psi\right)\left(\bar{a} C_{02}+\mu C_{11}\right) \\
\left(\bar{D}_{\dot{\alpha}} \bar{\Psi}\right)\left(a \tilde{C}_{02}-\mu \tilde{C}_{11}\right)
\end{array}\right) .
\end{aligned}
$$


Using $\left(D^{\alpha} \Psi\right) \mu=\frac{1}{2} a\left(\bar{D}_{\dot{\alpha}} \bar{\Psi}\right) \partial^{\dot{\alpha} \alpha}$ and $\left(\bar{D}_{\dot{\alpha}} \bar{\Psi}\right) \mu=-\frac{1}{2} \bar{a}\left(D^{\alpha} \Psi\right) \partial_{\alpha \dot{\alpha}}$ we can split the above to get the second order DE for $\check{B}_{2}$

$$
\left(\frac{\mathrm{d}^{2}}{\mathrm{~d} s^{2}}+u^{2}\right)\left(\begin{array}{c}
\check{B}_{2} \\
\check{\tilde{B}}_{2}
\end{array}\right)=\mathrm{i}\left(\begin{array}{cc}
0 & \Psi \\
\bar{\Psi} & 0
\end{array}\right)\left(\begin{array}{c}
C_{02} \\
\tilde{C}_{02}
\end{array}\right)-\frac{\mathrm{i}}{2} \frac{\mathrm{d}}{\mathrm{d} s}\left(\begin{array}{c}
C_{11} \\
\tilde{C}_{11}
\end{array}\right),
$$

the solution of which immediately gives $\hat{B}_{2}$ through the relation

$$
\left(\begin{array}{c}
\hat{B}_{2} \\
\tilde{\tilde{B}}_{2}
\end{array}\right)=\frac{-1}{\bar{\Psi} \Psi}\left(\begin{array}{cc}
0 & \Psi \\
\bar{\Psi} & 0
\end{array}\right)\left(\frac{\mathrm{d}}{\mathrm{d} s}\left(\begin{array}{c}
\check{B}_{2} \\
\check{\tilde{B}}_{2}
\end{array}\right)+\frac{\mathrm{i}}{2}\left(\begin{array}{c}
C_{11} \\
\tilde{C}_{11}
\end{array}\right)\right) .
$$

The final DE is easily read from the highest order terms in (A.9d),

$$
\left(\frac{\mathrm{d}^{2}}{\mathrm{~d} s^{2}}+2 i \beta \frac{\mathrm{d}}{\mathrm{d} s}+u^{2}\right)\left(\begin{array}{c}
C_{22} \\
\tilde{C}_{22}
\end{array}\right)=\frac{\square}{2} \frac{\mathrm{d}}{\mathrm{d} s}\left(\begin{array}{c}
\check{B}_{2} \\
\tilde{B}_{2}
\end{array}\right) .
$$

\section{A.2 Results for the heat kernel}

The differential equations (A.11) for $\left(C_{0}, \tilde{C}_{0}\right)$ are the only ones that are both homogeneous and have non-vanishing initial conditions. So, their integration is straightforward, with the results given in (A.23a). The DEs that need to be solved to find the terms of higher order in $D_{a} \Psi$ and $\bar{D}_{\dot{\alpha}} \bar{\Psi}$ all second order, inhomogeneous DEs with vanishing initial conditions. That is, they are all of the form

$$
\begin{aligned}
\left(\frac{\mathrm{d}^{2}}{\mathrm{~d} s^{2}}+2 \mathrm{i} \beta \frac{\mathrm{d}}{\mathrm{d} s}+u^{2}\right) \chi_{C}(s) & =v_{C}(s), & \chi_{C}(0) & =\dot{\chi}_{C}(0)=0, \\
\left(\frac{\mathrm{d}^{2}}{\mathrm{~d} s^{2}}+u^{2}\right) \chi_{B}(s) & =v_{B}(s), & \chi_{B}(0) & =\dot{\chi}_{B}(0)=0,
\end{aligned}
$$

where the $\chi_{B, C}$ are component 2-vectors of $B$ or $C$ respectively and the inhomogeneous terms $v_{B, C}$ depend on the solutions to lower order components. Using variation of parameters on the general solutions to the associated homogeneous differential equations yields

$$
\begin{aligned}
& \chi_{C}(s)=\mathrm{e}^{\mathrm{i} s \beta(\mathcal{G} / \mathcal{F}-1)} \int_{0}^{s} \mathrm{~d} t \mathrm{e}^{-2 \mathrm{i} t \beta \mathcal{G} / \mathcal{F}} \int_{0}^{t} \mathrm{~d} \tau \mathrm{e}^{\mathrm{i} \tau \beta(\mathcal{G} / \mathcal{F}+1)} v_{C}(\tau), \\
& \chi_{B}(s)=\mathrm{e}^{\mathrm{i} s u} \int_{0}^{s} \mathrm{~d} t \mathrm{e}^{-2 \mathrm{i} t u} \int_{0}^{t} \mathrm{~d} \tau \mathrm{e}^{\mathrm{i} \tau u} v_{B}(\tau) .
\end{aligned}
$$

The following solutions have all been found by hand and checked that they satisfy the original DEs and boundary conditions using Mathematica. The solutions for the components of $(C(s), \tilde{C}(s))$ are

$$
\begin{aligned}
& \left(\begin{array}{l}
C_{0} \\
\tilde{C}_{0}
\end{array}\right)=-\mathrm{i} \frac{\sin (s \mathcal{G})}{\mathcal{G}} \mathrm{e}^{-\mathrm{i} s \beta}\left(\begin{array}{c}
\bar{\Psi} \\
\Psi
\end{array}\right), \\
& \left(\begin{array}{l}
C_{11} \\
\tilde{C}_{11}
\end{array}\right)=\frac{s}{8 \mathcal{F}^{2}}\left(\frac{\sin (s u)}{s u}-\frac{\sin (s \mathcal{G})}{s \mathcal{G}} \cos (s \mathcal{F})\right)\left(\begin{array}{l}
\bar{\Psi} \\
\Psi
\end{array}\right),
\end{aligned}
$$




$$
\begin{aligned}
& \left(\begin{array}{l}
C_{20} \\
\tilde{C}_{20}
\end{array}\right)=\frac{\square \beta}{8 \mathcal{F}^{2}}\left(\begin{array}{cc}
0 & \Psi \\
\bar{\Psi} & 0
\end{array}\right)\left[\frac{\mathrm{i} s}{2 u^{2}}\left(\frac{\sin (s \mathcal{F})}{s \mathcal{F}} \cos (s \mathcal{G})-\cos (s \mathcal{F}) \frac{\sin (s \mathcal{G})}{s \mathcal{G}}\right)\right. \\
& +\frac{\beta}{u^{2}}\left(\frac{\cos (s u)}{\mathcal{F}^{2}}-\frac{\sin (s \mathcal{F}) \sin (s \mathcal{G})}{\mathcal{F} \mathcal{G}}-\frac{\cos (s \mathcal{F}) \cos (s \mathcal{G})}{\mathcal{F}^{2}}\right) \\
& \left.-\frac{\mathrm{i} s}{2 \mathcal{G}^{2}}\left(\cos (s \mathcal{G})-\frac{\sin (s \mathcal{G})}{s \mathcal{G}}\right) \mathrm{e}^{-\mathrm{i} s \beta}\right]\left(\begin{array}{l}
\bar{\Psi} \\
\Psi
\end{array}\right), \\
& \left(\begin{array}{l}
C_{02} \\
\tilde{C}_{02}
\end{array}\right)=\frac{\square}{16}\left(\begin{array}{cc}
0 & \Psi \\
\bar{\Psi} & 0
\end{array}\right) \frac{-\mathrm{i} \beta}{\mathcal{F}^{2}}\left[\frac{\sin (s \mathcal{G}) \cos (s \mathcal{F})}{u^{2} \mathcal{G}}-\frac{\sin (s \mathcal{F}) \cos (s \mathcal{G})}{u^{2} \mathcal{F}}\right. \\
& \left.+\frac{s \mathrm{e}^{-\mathrm{i} s \beta}}{\mathcal{G}^{2}}\left(\cos (s \mathcal{G})-\frac{\sin (s \mathcal{G})}{s \mathcal{G}}\right)\right]\left(\begin{array}{l}
\bar{\Psi} \\
\Psi
\end{array}\right), \\
& \left(\begin{array}{l}
C_{22} \\
\tilde{C}_{22}
\end{array}\right)=\frac{-\mathrm{i} \square}{128 \mathcal{F}^{2}}\left[\frac{\mathrm{i} s^{2} \beta}{\mathcal{F}^{2}}\left(\frac{\sin (s \mathcal{F})}{s \mathcal{F}} \frac{\sin (s \mathcal{G})}{s \mathcal{G}}-\frac{\sin (s u)}{s u}\right)+s \mathrm{e}^{-\mathrm{i} s \beta} \times\right. \\
& \times\left(\frac{\sin (s \mathcal{G})}{s \mathcal{G}}\left(\frac{1+\mathrm{i} s \beta}{\mathcal{F}^{2}}-\frac{3 \mathcal{F}^{2}+\left(1+s^{2} u^{2}\right) \mathcal{G}^{2}}{2 \mathcal{G}^{4}}\right)+\cos (s \mathcal{G}) \frac{3 \mathcal{F}^{2}+\mathcal{G}^{2}}{2 \mathcal{G}^{4}}\right) \\
& \left.-\frac{s}{u^{2}}\left(\frac{u^{2}}{\mathcal{F}^{2}} \frac{\sin (s u)}{s u}+\frac{\sin (s \mathcal{F})}{s \mathcal{F}} \cos (s \mathcal{G})-\cos (s \mathcal{F}) \frac{\sin (s \mathcal{G})}{s \mathcal{G}}\right)\right]\left(\begin{array}{l}
\bar{\Psi} \\
\Psi
\end{array}\right),
\end{aligned}
$$

and the solutions for the components of $\left(B^{\alpha}(s), \tilde{B}_{\dot{\alpha}}(s)\right)$ are

$$
\begin{aligned}
& \left(\begin{array}{c}
\check{B}_{0} \\
\check{\tilde{B}}_{0}
\end{array}\right)=\frac{\mathrm{i} s}{2 u^{2}}\left(\begin{array}{cc}
0 & \Psi \\
\bar{\Psi} & 0
\end{array}\right)\left(\frac{\beta}{\mathcal{F}} \frac{\cos (s u)}{s \mathcal{F}}-\mathrm{e}^{-\mathrm{i} s \beta}\left(\mathrm{i} \frac{\sin (s \mathcal{G})}{s \mathcal{G}}+\frac{\beta}{\mathcal{F}} \frac{\cos (s \mathcal{G})}{s \mathcal{F}}\right)\right)\left(\begin{array}{l}
\bar{\Psi} \\
\Psi
\end{array}\right), \\
& \left(\begin{array}{c}
\hat{B}_{0} \\
\hat{\tilde{B}}_{0}
\end{array}\right)=\frac{\mathrm{i} s \beta}{2 \mathcal{F}^{2}}\left(\frac{\sin (s u)}{s u}-\frac{\sin s \mathcal{G}}{s \mathcal{G}} \mathrm{e}^{-\mathrm{i} s \beta}\right)\left(\begin{array}{c}
\bar{\Psi} \\
\Psi
\end{array}\right), \\
& \left(\begin{array}{c}
\check{B}_{2} \\
\check{\tilde{B}}_{2}
\end{array}\right)=\frac{-\mathrm{i} s^{2}}{32 \mathcal{F}^{2}}\left[\frac{2 \mathrm{i} \beta}{s u^{2}}\left(\frac{\mathcal{G}^{2}}{\mathcal{F}^{2}} \frac{\sin (s \mathcal{G})}{s \mathcal{G}} \cos (s \mathcal{F})-\cos (s \mathcal{G}) \frac{\sin (s \mathcal{F})}{s \mathcal{F}}\right)\right. \\
& \left.+\frac{\sin (s u)}{s u}\left(1-\frac{2 \mathrm{i} \beta}{s \mathcal{F}^{2}}\right)-\mathrm{e}^{-\mathrm{i} s \beta}\left(\left(1+\frac{\mathrm{i} \beta}{s \mathcal{G}^{2}}\right) \frac{\sin (s \mathcal{G})}{s \mathcal{G}}-\frac{\mathrm{i} \beta}{\mathcal{G}} \frac{\cos (s \mathcal{G})}{s \mathcal{G}}\right)\right]\left(\begin{array}{l}
\bar{\Psi} \\
\Psi
\end{array}\right), \\
& \left(\begin{array}{c}
\hat{B}_{2} \\
\hat{\tilde{B}}_{2}
\end{array}\right)=\frac{-\mathrm{i}}{\bar{\Psi} \Psi} \frac{1}{16 \mathcal{F}^{2}}\left(\begin{array}{cc}
0 & \Psi \\
\bar{\Psi} & 0
\end{array}\right)\left[\frac{\sin (s u)}{2 u}-\cos (s u) \frac{s \mathcal{F}^{2}-2 \mathrm{i} \beta}{2 \mathcal{F}^{2}}\right. \\
& -\left(\frac{\sin (s \mathcal{G})}{\mathcal{G}}+\frac{\mathrm{i} \beta}{\mathcal{F}^{2}} \cos (s \mathcal{G})\right) \cos (s \mathcal{F}) \\
& \left.+\frac{1}{2 \mathcal{G}^{2}}\left(u^{2} s \cos (s \mathcal{G})+\left(\mathcal{F}^{2}+\mathcal{G}^{2}\right) \frac{\sin (s \mathcal{G})}{\mathcal{G}}\right) \mathrm{e}^{-\mathrm{i} s \beta}\right]\left(\begin{array}{l}
\bar{\Psi} \\
\Psi
\end{array}\right) \text {. }
\end{aligned}
$$

The solution for $(A, \tilde{A})$ is just a term-by-term integration (A.9a) of the expression for $(C, \tilde{C})$ given in (A.23) above.

From the above results, it is easily checked that the solutions satisfy the initial condition $\Omega(0)=1$. They also satisfy the initial velocity condition $\Omega^{\prime}(0)=-\frac{i}{4}\left(\bar{\Psi} D^{2}+\Psi \bar{D}^{2}\right)$, which implies that only $\left(C_{0}, \tilde{C}_{0}\right)$ has a non-vanishing first derivative at $s=0$. 
The above results for the coefficients in the expansion of $\Omega(s)=$ $\exp \left(-\frac{i s}{4}\left(\bar{\Psi} D^{2}+\Psi \bar{D}^{2}\right)\right)$ combined with the factorisation

$$
U_{V}^{(\Psi)}\left(z, z^{\prime} \mid s\right)=\Omega(s) U_{V}^{(0)}\left(z, z^{\prime} \mid s\right), \quad U_{V}^{(0)}\left(z, z^{\prime} \mid s\right)=\delta^{4}\left(\theta-\theta^{\prime}\right) U\left(x, x^{\prime} \mid s\right),
$$

give the full solution for the heat kernel of the Wess-Zumino model in four dimensions in terms of the bosonic heat kernel (A.5). The dimensionally reduced heat kernel is obtained by simply replacing the momentum integral in (A.5) with its dimensionally reduced counterpart

$$
\begin{aligned}
U\left(x, x^{\prime} \mid s\right) & =\exp (\mathrm{i} s \square) \delta^{d}\left(x-x^{\prime}\right) \\
& =\mu^{2 \varepsilon} \int \frac{\mathrm{d}^{d} k}{(2 \pi)^{d}} \mathrm{e}^{-\mathrm{i} k^{2} s+\mathrm{i} k\left(x-x^{\prime}\right)}=\frac{\mathrm{i} \mu^{2 \varepsilon}}{(4 \pi \mathrm{i} s)^{d / 2}} \mathrm{e}^{\frac{\mathrm{i}}{4}\left(x-x^{\prime}\right)^{2} / s} .
\end{aligned}
$$

\section{A.3 Kähler approximation}

As a check on the general results above, we examine the limit of the heat kernel that is appropriate for computing the corrections to the Kähler potential. That is, we enforce the condition $\Psi=$ const by taking the limits as $a, b$, and $\mu$ go to 0 , which implies that $u^{2}=\mathcal{G}^{2}=\bar{\Psi} \Psi \square$ and $A=\tilde{A}=\bar{\Psi} \Psi(\cos (s u)-1) / u^{2}$. So, the expansion of $\Omega(s)$ reduces to

$$
\Omega(s)=1-\frac{\mathrm{i}}{4} \frac{\sin s u}{u}\left(\bar{\Psi} D^{2}+\Psi \bar{D}^{2}\right)+\frac{\bar{\Psi} \Psi}{16} \frac{\cos s u-1}{u^{2}}\left\{D^{2}, \bar{D}^{2}\right\}
$$

which matches that presented in [6-8].

This result can also be derived directly from (A.6). When $\Psi=$ const, we can take a second proper-time derivative to find the inhomogeneous harmonic oscillator equation ${ }^{7}$

$$
\Omega^{\prime \prime}(s)=-\frac{1}{16} \Omega(s)\left(\bar{\Psi} D^{2}+\Psi \bar{D}^{2}\right)^{2}=-u^{2} \Omega(s)\left(P_{+}+P_{-}\right)=-u^{2} \Omega(s)+u^{2} P_{0},
$$

with the initial conditions $\Omega(0)=1, \Omega^{\prime}(0)=-\frac{\mathrm{i}}{4}\left(\bar{\Psi} D^{2}+\Psi \bar{D}^{2}\right)$. This is easily solved to give (A.26).

Open Access. This article is distributed under the terms of the Creative Commons Attribution License (CC-BY 4.0), which permits any use, distribution and reproduction in any medium, provided the original author(s) and source are credited.

\section{References}

[1] J. Wess and B. Zumino, Supergauge transformations in four-dimensions, Nucl. Phys. B 70 (1974) 39 [inSPIRE].

[2] J. Wess and B. Zumino, A lagrangian model invariant under supergauge transformations, Phys. Lett. B 49 (1974) 52 [INSPIRE].

[3] Y. Golfand and E.P. Likhtman, Extension of the algebra of Poincaré group generators and violation of $p$ invariance, JETP Lett. 13 (1971) 323 [INSPIRE].

\footnotetext{
${ }^{7}$ In deriving this differential equation we used the superspace projection operators given in (2.16).
} 
[4] D.V. Volkov and V.P. Akulov, Possible universal neutrino interaction, JETP Lett. 16 (1972) 438 [Pisma Zh. Eksp. Teor. Fiz. 16 (1972) 621] [INSPIRE].

[5] D.V. Volkov and V.P. Akulov, Is the neutrino a Goldstone particle?, Phys. Lett. B 46 (1973) 109 [INSPIRE].

[6] I.L. Buchbinder and S.M. Kuzenko, Ideas and methods of supersymmetry and supergravity or a walk through superspace, IOP, Bristol U.K. (1995) [INSPIRE].

[7] I.L. Buchbinder, S.M. Kuzenko and Z. Yarevskaya, Superfield approach to the calculation of the effective potential in supersymmetric field theories, Phys. Atom. Nucl. 56 (1993) 680 [INSPIRE].

[8] I.L. Buchbinder, S. Kuzenko and Z. Yarevskaya, Supersymmetric effective potential: superfield approach, Nucl. Phys. B 411 (1994) 665 [INSPIRE].

[9] J. Iliopoulos and B. Zumino, Broken supergauge symmetry and renormalization, Nucl. Phys. B 76 (1974) 310 [inSPIRE].

[10] S. Ferrara, J. Wess and B. Zumino, Supergauge multiplets and superfields, Phys. Lett. B 51 (1974) 239 [inSPIRE].

[11] R. Delbourgo, Superfield perturbation theory and renormalization, Nuovo Cim. A 25 (1975) 646 [INSPIRE].

[12] P.C. West, The supersymmetric effective potential, Nucl. Phys. B 106 (1976) 219 [INSPIRE].

[13] S. Weinberg, Ambiguous solutions of supersymmetric theories, Phys. Lett. B 62 (1976) 111 [INSPIRE].

[14] M.T. Grisaru, W. Siegel and M. Roček, Improved methods for supergraphs, Nucl. Phys. B 159 (1979) 429 [inSPIRE].

[15] S.J. Gates, M.T. Grisaru, M. Roček and W. Siegel, Superspace or one thousand and one lessons in supersymmetry, Benjamin/Cummings, Reading U.S.A. (1983) [hep-th/0108200] [INSPIRE].

[16] P.C. West, A comment on the nonrenormalization theorem in supersymmetric theories, Phys. Lett. B 258 (1991) 375 [INSPIRE].

[17] I. Jack, D.R.T. Jones and P.C. West, Not the no-renormalization theorem?, Phys. Lett. B 258 (1991) 382 [InSPIRE].

[18] I.L. Buchbinder, S.M. Kuzenko and A.Y. Petrov, Superfield chiral effective potential, Phys. Lett. B 321 (1994) 372 [inSPIRE].

[19] K. Fujikawa and W. Lang, On the effective potential for the scalar multiplet in the supersymmetric $\phi^{3}$ model, Nucl. Phys. B 88 (1975) 77 [INSPIRE].

[20] L. O'Raifeartaigh and G. Parravicini, Effective potential for chiral scalar superfields, Nucl. Phys. B 111 (1976) 516 [inSPIRE].

[21] M. Huq, Evaluation of effective potential in superspace, Phys. Rev. D 16 (1977) 1733 [INSPIRE].

[22] D. Amati and K.-c. Chou, On the determination of effective potentials in supersymmetric theories, Phys. Lett. B 114 (1982) 129 [INSPIRE].

[23] M.T. Grisaru, F. Riva and D. Zanon, The one loop effective potential in superspace, Nucl. Phys. B 214 (1983) 465 [inSPIRE]. 
[24] R.D.C. Miller, A simple component field method for SUSY effective potential calculations, Phys. Lett. B 124 (1983) 59 [InSPIRE].

[25] R.D.C. Miller, A tadpole supergraph method for the evaluation of SUSY effective potentials, Nucl. Phys. B 228 (1983) 316 [inSPIRE].

[26] G. Fogleman, G.D. Starkman and K.S. Viswanathan, Two loop calculation of the effective potential for the Wess-Zumino model, Phys. Lett. B 133 (1983) 393 [INSPIRE].

[27] A. Pickering and P.C. West, The one loop effective superpotential and nonholomorphicity, Phys. Lett. B 383 (1996) 54 [hep-th/9604147] [INSPIRE].

[28] N.G. Pletnev and A.T. Banin, Covariant technique of derivative expansion of one loop effective action. 1, Phys. Rev. D 60 (1999) 105017 [hep-th/9811031] [INSPIRE].

[29] M.T. Grisaru, M. Roček and R. von Unge, Effective Kähler potentials, Phys. Lett. B 383 (1996) 415 [hep-th/9605149] [INSPIRE].

[30] B. de Wit, M.T. Grisaru and M. Roček, Nonholomorphic corrections to the one loop $N=2$ super Yang-Mills action, Phys. Lett. B 374 (1996) 297 [hep-th/9601115] [INSPIRE].

[31] S. Groot Nibbelink and T.S. Nyawelo, Two loop effective Kähler potential of (non-)renormalizable supersymmetric models, JHEP 01 (2006) 034 [hep-th/0511004] [INSPIRE].

[32] S.J. Tyler, Studies of low-energy effective actions in supersymmetric field theories, Ph.D. Thesis, University of Western Australia, Australia (2013), arXiv:1401.4814 [INSPIRE].

[33] A. Salam and J.A. Strathdee, On superfields and Fermi-Bose symmetry, Phys. Rev. D 11 (1975) 1521 [inSPIRE].

[34] E. Sokatchev, Projection operators and supplementary conditions for superfields with an arbitrary spin, Nucl. Phys. B 99 (1975) 96 [inSPIRE].

[35] A.T. Banin, I.L. Buchbinder and N.G. Pletnev, Effective action in $N=2,4$ supersymmetric Yang-Mills theories, Grav. Cosmol. 9 (2003) 2 [hep-th/0309048] [INSPIRE].

[36] R.D.C. Miller, The two loop effective potential of the Wess-Zumino model, Nucl. Phys. B 241 (1984) 535 [INSPIRE].

[37] G. Fogleman and K.S. Viswanathan, The effective potential for chiral supersymmetric models, Phys. Rev. D 30 (1984) 1364 [INSPIRE].

[38] R. dos Santos and P. Srivastava, Two-loop effective potential for Wess-Zumino model using superfields, Rev. Bras. Fis. 19 (1989) 7 [INSPIRE]. 